Research International

Elsevier Editorial System(tm) for Food

Manuscript Draft

Manuscript Number: FOODRES-D-17-01325R1

Title: Effect of chemical composition and sonication procedure on properties of food-grade soy lecithin liposomes with added glycerol

Article Type: Research Paper

Keywords: soy lecithin, lipid fractionation, phospholipids, liposomes, sonication, glycerol

Corresponding Author: Dr. M.Carmen Gómez-Guillén,

Corresponding Author's Institution:

First Author: Diego Taladrid

Order of Authors: Diego Taladrid; Daniel Marín; Ailén Alemán; Inmaculada Álvarez-Acero; Pilar Montero; M.Carmen Gómez-Guillén

Abstract: The effect of two-step and five-step acetone washing of soybean lecithin (SL) on compositional properties of partially purified phosphatidylcholines (PW2 and PW5) was studied. Trace amounts of protein were detected in SL, PW2 and PW5, with a predominance of glutamic acid and aspartic acid. Increasing the number of acetone washing steps significantly reduced the total content of $\gamma^{-}, \delta-$ and $\alpha$-tocopherol. Similar reductions $(\approx 90 \%)$ of neutral lipids were found in both PW2 and PW5, but the removal of free fatty acids was higher in PW5 than in PW2 (78\% vs. 71\%). Linoleic acid was the main constituent in both the neutral lipids and the phospholipid fractions of SL, PW2 and PW5, accounting for around 53-59\% of total fatty acids; however, a considerable amount of it was removed from the free fatty acid fraction by increasing the number of washing steps. All phospholipid classes were mostly concentrated in the first two-step washing of lecithin. Further washing increased the concentrations of phosphatidylcholine (PC), phosphatidylethanolamine (PE), lyso-PC and lyso-PE in PW5, as compared to PW2. Glycerol-containing liposomes from PW2 and PW5 were produced using two different-intensity sonication procedures (A: 90\% amplitude, 5 min; B: 20\% amplitude, 2 min). Liposomes of soy lecithin and liposomes of PW5 without glycerol were also obtained by using strong sonication (method A). The liposomal dispersion with the highest purification and the stronger sonication was clearly distinguished from the others, both in particle size and in zeta potential. DSC results showed noticeable interference of glycerol in the membrane structure, but minimal changes in particle size and surface charge. 


\section{Effect of chemical composition and sonication procedure on properties of food-grade soy lecithin liposomes with added glycerol.}

D. Taladrid, D. Marín, A. Alemán, I. Álvarez-Acero, P. Montero, M.C. Gómez-Guillén*

Institute of Food Science, Technology and Nutrition (ICTAN-CSIC)

José Antonio Novais 10, 28040 Madrid (Spain)

*Author for correspondence: $\underline{\text { mc.gomez@csic.es }}$

(1)

\section{ABSTRACT}

The effect of two-step and five-step acetone washing of soybean lecithin (SL) on compositional properties of partially purified phosphatidylcholines (PW2 and PW5) was studied. Trace amounts of protein were detected in SL, PW2 and PW5, with a predominance of glutamic acid and aspartic acid. Increasing the number of acetone washing steps significantly reduced the total content of $\psi^{-}, \delta$ - and $\alpha$-tocopherol. Similar reductions $(\approx 90 \%)$ of neutral lipids were found in both PW2 and PW5, but the removal of free fatty acids was higher in PW5 than in PW2 (78\% vs. $71 \%)$. Linoleic acid was the main constituent in both the neutral lipids and the phospholipid fractions of SL, PW2 and PW5, accounting for around $53-59 \%$ of total fatty acids; however, a considerable amount of it was removed by increasing the number of washing steps. All phospholipid classes were mostly concentrated in the first two-step washing of lecithin. Further washing increased the concentration of phosphatidylcholine (PC) in PW5, as compared to PW2. Glycerol-containing liposomes from PW2 and PW5 were produced using two different-intensity sonication procedures (method A: 120W, 5 min; method B: 30W, 2 min) using a probe-type sonicator (100 $\mathrm{mL}$ volume suspension). Liposomes of soy lecithin and liposomes of PW5 without glycerol were also obtained by using strong sonication (method A). The liposomal dispersion with the highest purification and the stronger sonication was clearly 
25 distinguished from the others, both in particle size and in zeta potential. DSC results showed

26 noticeable interference of glycerol in the membrane structure, but minimal changes in particle 27 size and surface charge.

28

29 Keywords: soy lecithin, lipid fractionation, phospholipids, liposomes, sonication, glycerol 
31 Liposomes are typically spherical lipid vesicles consisting of one or more phospholipid bilayers that have the ability to entrap a volume of aqueous medium. They can be used as delivery carriers for both lipophilic and hydrophilic compounds, owing to their amphiphilic structure and composition. Liposomes have been extensively studied as a model membrane system, owing to their strong resemblance to biological cell membranes (Mozafari, Johnson, Hatziantoniou \& Demetzos, 2008). Liposome properties differ considerably with regard to lipid composition, surface charge, size and method of preparation (Akbarzadeh et al., 2013). The compositional, physicochemical and structural properties of liposomes are important factors determining their aptitude in potential pharmaceutical, clinical and food applications. In the food industry, they are used as flavour encapsulators and taste maskers, and as carriers of vitamins, antioxidants, proteins, enzymes and minerals, protecting compounds from interaction with other food matrix components and providing enhanced stability against chemical or physical degradation (Da Silva Malheiros, Daroit \& Brandelli, 2010).

Liposomes can be prepared from a variety of lipids. Many studies have been carried out with synthetic phosphatidylcholines, such as dipalmitoylphosphatidylcholine (DPPC) and dimyristoylphosphatidylcholine (DMPC), with a well-defined saturated fatty acid and polar head group composition, since they produce stable and well-packed bilayer membranes (Li et al., 2015). The use of non-synthetic lecithin, such as soy lecithin, for liposome production does not raise any food legislation concerns and provides nutritional value owing to the high polyunsaturated fatty acid composition (Laye, McClements \& Weiss, 2008). However, the predominance of highly unsaturated fatty acids gives typically more permeable and less stable bilayers (Biltonen \& Lichtenberg, 1993), and may eventually generate hydroperoxides and secondary oxidation products, whose accumulation in a food system could give rise to rancidity and a concomitant shelf life reduction. To avoid this drawback, the production of 

undesirable oxidation products (Frenzel \& Steffen-Heins, 2015).

57 be admissible for most food applications. 2010). (Manca et al., 2013).

liposomes loaded with antioxidant compounds such as quercetin may reduce the formation of

The presence of impurities in natural lecithin can also influence the properties and stability of derived liposomes. The phospholipids can be purified by successive extractions with organic solvents, of which acetone is one of the most commonly used; however, variable amounts of non-phospholipid components, such as triglycerides, free fatty acids, sterols, pigments and carbohydrates, may remain (Van Hoogevest \& Wendel, 2014). The presence of impurities could be a serious impediment for parenteral liposome-based drug administration, but it could

Liposomes can be obtained in a range of sizes, from a few nanometres to several micrometres. Among the various liposome-producing methods, sonication is one of the most widely used for small unilamellar vesicle (SUV) preparation; however, the very high energy input into lipid dispersion may induce some phospholipid degradation (Silva, Ferreira, Little \& Cavaco-Paulo,

Glycerol has been extensively studied as a cryoprotectant to provide increased liposomal stabilization in freezing and/or drying processes (Stark, Pabst \& Prassl, 2010). This property could be of great importance for potential food applications, such as frozen functional foods or dried functional edible films (Alemán et al., 2016). In addition, the use of glycerol for liposome preparation could further expand the range of applications by modifying membrane fluidity

The aim of this work was to study the effect of two-step and five-step acetone washing of soybean lecithin (SL) on compositional properties of partially purified phosphatidylcholines (PW2 and PW5), and their influence on the hydrodynamic properties, membrane surface charge and structural properties of the corresponding glycerol-containing liposomes, which were produced by comparing two sonication procedures of different intensities [method A: 

purposes, liposomes made of soy lecithin and liposomes made of PW5 without glycerol were also obtained, using strong sonication (method A).

\section{Material and methods}

\subsection{Raw materials and reagents}

Soybean lecithin (SL) was obtained from Manuel Riesgo S.A. (Madrid, Spain). A triglyceride formed by three 13:0 chains (T3882) and a 19:0 free fatty acid (N5252) (Sigma-Aldrich, St. Louis, MO, USA) were employed as standards for determination of the fatty acid profile of the fraction of neutral lipids (NL) and free fatty acids (FFA), respectively, while a phospholipid formed by two 15:0 chains (Cymit Química S.L., Barcelona, Spain) was used for the fraction corresponding to phospholipids (PL). In the tocopherol assay, the following standards were used: DL-Alpha Tocopherol 47783 Supelco, Delta Tocopherol 47784 Supelco and Gamma Tocopherol 47785 Supelco (Sigma-Aldrich). Norleucine was employed as an internal pattern in the determination of the amount of each amino acid.

\subsection{Phospholipid extraction}

Two partially purified phosphatidylcholine powders were obtained from soybean lecithin following the procedure described by Mosquera et al. (2014). The difference between them was the number of washing steps with acetone: two washes (PW2) or five washes (PW5). Commercial soybean lecithin (Manuel Riesgo, S.A., Madrid, Spain) was dissolved in ethyl acetate $(1: 5, w / v)$. Then, distilled water was added slowly and under agitation, until two phases were formed. The upper liquid phase was discarded. The phospholipid extraction from

102 the lower phase was performed using two or fives washes with acetone $(1: 2 \mathrm{w} / \mathrm{v})$, being the acetone separated by decanting. The precipitate was vacuum-filtered, dried in a desiccator 
during $24 \mathrm{~h}$ at $24^{\circ} \mathrm{C}$ in darkness and ground to obtain a fine powder. The partially purified phosphatidylcholine powders [PW2 (2 washes) and PW5 (5 washes)] were stored at $-20^{\circ} \mathrm{C}$ until use.

\subsection{Chemical composition of the raw materials}

Crude lipid content was determined by the method of Bligh and Dyer (1959), basically consisting in homogenizing the raw samples with dichloromethane, methanol and water, allowing to form two phases. The upper phase was discarded. The lower phase was rotaevaporated at $60{ }^{\circ} \mathrm{C}$ and further dried at $105{ }^{\circ} \mathrm{C}$ until constant weight. The lipid content was calculated by weight differences with respect to the initial sample weight and expressed as a percentage. Measurements were carried out in triplicate.

Moisture was determined at least in triplicate according to the method 950.46 (AOAC, 2005). The samples were dried at $105{ }^{\circ} \mathrm{C}$ during $24 \mathrm{~h}$ and the water content was calculated by weight differences and expressed as a percentage.

Ash content was determined at least in triplicate according to the method 900.02A (AOAC, 2005). The samples were burnt at $500{ }^{\circ} \mathrm{C}$ during $24 \mathrm{~h}$ in a muffle furnace (Select-Horn, PSELECTA, EQ-056-CAM) and the ash content was calculated by weight differences and expressed as a percentage.

Protein content was determined from the amino acid composition, as described previously (Alemán, Gómez-Guillén \& Montero, 2013). The samples were suspended at $1 \mathrm{mg} / \mathrm{mL}$ in MiliQ water. An amount of $50 \mu \mathrm{L}$ of sample were dried and hydrolyzed in vacuum-sealed glass tubes at $110^{\circ} \mathrm{C}$ for $24 \mathrm{~h}$ in the presence of continuously boiling $6 \mathrm{~N} \mathrm{HCl}$ containing $0.1 \%$ phenol with norleucine (Sigma-Aldrich, Inc., St. Louis, MO, USA) as internal standard. After hydrolysis, samples were again vacuum-dried, dissolved in application buffer, and injected onto a 
127 Biochrom 20 amino acid analyzer (Pharmacia, Barcelona, Spain). Results are average of three 128 determinations.

129

130

131

132

133

134

135

136

\subsubsection{Fatty acid profile of different lipid fractions}

Firstly, raw samples (SL, PW2 and PW5) were separated into three main lipid fractions (Neutral lipids, NL; Free fatty acids, FFA, and Phospholipids, $P L$ ), and then the fatty acid composition was determined for each lipid fraction. SL, PW2 and PW5 were dissolved in hexane $(0.133$ $\mathrm{mg} / \mathrm{mL}$ ). The fractionation was initiated from $500 \mu \mathrm{L}$ of this solution in SPE using SPE Bond Elut NH2 200 mg columns (Agilent Technologies). Fractionation was carried out using positive pressure with a syringe by consecutive elution with a first phase of chloroform-isopropanol for $\mathrm{NL}$, a second phase of diethyl ether-acetic acid $2 \%$ for FFA, and a third phase of methanol-HCL 2\% for the phospholipid fraction (PL) (Álvarez et al., 2009). Each fraction was dried under nitrogen and derivatized to obtain fatty acid methyl esters (FAMEs) (Gómez-Estaca, Calvo, Álvarez-Acero, Montero \& Gómez-Guillén, 2017). FAMEs were injected (1 $\mu \mathrm{L})$ in a gas chromatograph with FID $260{ }^{\circ} \mathrm{C}$ detector (Agilent Technologies, Santa Clara, CA, USA), with $\mathrm{H}_{2}$ flow of $40 \mathrm{~mL} / \mathrm{min}$ and air flow of $450 \mathrm{~mL} / \mathrm{min}$. An HP88 $60 \mathrm{~m} \times 0.25 \mathrm{~mm} \times 0.2 \mu \mathrm{m}$ column (Agilent) was used. Samples were subjected to an initial temperature of $125{ }^{\circ} \mathrm{C}$ with subsequent ramps of $8{ }^{\circ} \mathrm{C} / \mathrm{min}$ up to $145^{\circ} \mathrm{C}, 2^{\circ} \mathrm{C} / \mathrm{min}$ up to $220^{\circ} \mathrm{C}$ and $10^{\circ} \mathrm{C} / \mathrm{min}$ to $125^{\circ} \mathrm{C}$. Analysis of data was carried out by means of the EZChrom Elite computer software (Agilent Technologies). Identification was accomplished by comparison of the retention times with the standards used. For quantification, an internal standard (C13:0) was used and results were expressed as $\mathrm{mg} / \mathrm{g}$ raw sample. Results are average of three determinations.

\subsubsection{Identification of phospholipids classes by LCMS}

Phospholipid molecular species identification was performed following the procedure described by Yan, Li, Xu, \& Zhou (2010) with some modifications. One millilitre of solution of $\mathrm{SL}$, PW2 and PW5 in chloroform $(65 \mathrm{mg} / \mathrm{mL})$ was evaporated with nitrogen, resuspended in 1 
$\mathrm{mL}$ of methanol and diluted 200-fold. Samples $(1 \mu \mathrm{L})$ were injected in an Agilent 1200 liquid chromatograph with diode-array detector, coupled to an Agilent Accurate-Mass QTOF-LC/MS, equipped with an ESI source (Agilent Technologies, Palo Alto, CA, USA). A Zorbax Eclipse XDBC8 column (4.6 mm x $150 \mathrm{~mm} \times 5 \mu \mathrm{m}$, Agilent Technologies), with $\mathrm{H}_{2} \mathrm{O}$-ammonium formate 5 $\mathrm{mM}$ as phase $\mathrm{A}$ and methanol as phase $\mathrm{B}$ (flow of $1 \mathrm{~mL} / \mathrm{min}$ at $30^{\circ} \mathrm{C}$ ), was used. Determinations were performed in duplicate both in positive-ion and negative-ion mode.

For estimation of PL composition, all possible combinations between seven groups of phospholipid classes [phosphatidylcholine (PC), phosphatidylethanolamine (PE), phosphatidylserine (PS), phosphatidylinositol (PI), phosphatidic acid (PA), lyso-PC and lysoPE] and the five major fatty acids previously found (18:2n6c, C18:1n9c, C16:0, C18:3n3 and C18:0) were considered, which made a total of 85 possible compounds. The mass spectra that were obtained were analysed by matching the theoretical mass of the formula and the exact measured mass (as positive $(\mathrm{M}+\mathrm{H})$ and $\left(\mathrm{M}+\mathrm{NH}_{4}\right)$, and negative $(\mathrm{M}-\mathrm{H})$ ions), employing the Masshunter Quantitative Analysis B.07.00 computer software (Agilent Technologies). From the 85 probable combinations, a total of 41 compounds were identified in SL, PW2 and PW5, their exact match formula being provided in Table 1. Results of phospholipid class composition were expressed in terms of signal intensity by considering the sum of all identified phospholipids within each class.

\subsubsection{Tocopherol composition}

SL, PW2 and PW5 samples were subjected to saponification and subsequent extraction with chloroform. An aliquot was dried and dissolved in methanol and then injected in an Agilent 1200 liquid chromatograph coupled to an Agilent Triple Quadrupole G6410 with an APCI source equipped with a Supelcosil LC-F column with pre-column (Gómez-Estaca et al., 2017). The mobile phase used was isocratic $100 \%$ methanol at a flow rate of $0.6 \mathrm{~mL} / \mathrm{min}$. A Supelcosil LC-F (4.6 mm $\times 150 \mathrm{~mm} \times 5 \mu \mathrm{m}$, Supelco) was used. The injection volume was $2 \mu \mathrm{L}$, in negative 
177 polarity. Identification was accomplished by comparison with the corresponding standards of

178 DL- $\alpha$-tocopherol (RT $5.61 \mathrm{~min}$ ), $\delta$-tocopherol (RT $5.47 \mathrm{~min}$ ) and -tocopherol (RT $5.44 \mathrm{~min}$ )

179 (Sigma-Aldrich), which were subjected to the same preparation procedure (saponification and subsequent extraction with chloroform) as the raw samples. For quantification, DL- $\alpha$ tocopherol was analysed as $\mathrm{MRM}^{-} 429.4$ to 163.1 , $\delta$-tocopherol as $\mathrm{MRM}^{-} 415.36$ to 149.1 and -tocopherol as $\mathrm{MRM}^{-} 429.4$ to 163.1 , using the Masshunter Qualitative Analysis B.07.00 computer software (Agilent Technologies). The quantification was performed by comparing the corresponding areas with those of the standards, using calibration curves in the concentration range between 0.04 and $2.5 \mu \mathrm{g} / \mathrm{mL}$. Results are average of three determinations.

\subsection{Infrared spectroscopy (ATR-FTIR)} Infrared Spectrometer (Perkin Elmer Inc, Waltham, MA, USA) equipped with an ATR prism crystal accessory. For each spectrum 32 scans of interferograms were averaged and the spectral resolution was $4 \mathrm{~cm}^{-1}$. Background was subtracted using the Spectrum software version 6.3.2 (Perkin Elmer Inc.). Measurements were performed at room temperature using a thin layer of the freeze-dried liposomal paste, which was placed on the surface of the ATR crystal, and pressed with a flat-tip plunger until spectra with suitable and stable peaks were obtained. All experiments were performed at least in triplicate.

\subsection{Liposome preparation}

Six types of liposomal suspensions were produced according to the method described by Alemán et al. (2016), with slight modifications consisting in the variation of these two parameters: raw material (SL, PW2 and PW5) and sonication method (strong: A; light: B) (Table 2). All liposomal suspensions were prepared with addition of glycerol $(3 \% \mathrm{w} / \mathrm{v})$, with the exception of $L 5 n g$, for comparison purposes. 
The sonication was performed using a Q700 sonicator (Qsonica, Newton, CT, EEUU, max 700 W). To sonicate $100 \mathrm{~mL}$ of liposomal suspension, a 1/2" diameter probe with a 1/2" (12.7 mm) replaceable tip was employed. The probe was introduced up to the middle of the suspension (leaving $2 \mathrm{~cm}$ above and $2 \mathrm{~cm}$ below). Method A (strong) consisted in sonicating with intensity set at $90 \%$ (measured power $120 \mathrm{~W}$ ) for 5 minutes in a pulsed mode, with a $60 \mathrm{~s}$ stop every min to allow sample cooling. Method B (light) consisted in sonicating with intensity set at $20 \%$ (measured power $30 \mathrm{~W}$ ) for $2 \mathrm{~min}$, with the same intervals as above. All suspensions were sonicated surrounded by crushed ice to avoid overheating. Maximum temperature did not exceed $70^{\circ} \mathrm{C}$ in any case.

\subsection{Characterization of nanovesicles}

\subsubsection{Size, polydispersity and zeta potential}

Particle size, polydispersity and zeta potential were determined by using a Zetasizer Nano ZS (Malvern Instruments Ltd, Worcestershire, UK). Measurements were carried out in triplicate at $25^{\circ} \mathrm{C}$ after 10 -fold dilution of the liposomal suspensions in distilled water, as reported earlier (Alemán et al., 2016). The mean particle size, expressed a z-average value, was calculated using the Malvern Zetasizer Software PSS0012-38 v7.12, following the Stokes-Einstein equation:

where $D z$ is the hydrodynamic diameter; $D t, a v g$ is the translational diffusion coefficient (by DLS); $k_{B}$ is Boltzmann's constant; $T$ is thermodynamic temperature and $\eta$ is dynamic viscosity.

\subsubsection{Thermal properties}


224 scanning calorimeter (DSC) (TA Instruments, New Castle, DE, USA) previously calibrated by running high purity indium (melting point, $156.4{ }^{\circ} \mathrm{C}$; melting enthalpy, $28.44 \mathrm{~J} / \mathrm{g}$ ). Samples of 1 $\mathrm{mL}$ of each fresh liposomal suspension $(6 \mathrm{mg} / \mathrm{mL})$ were concentrated using a SpeedVac system

227 (Thermo Fisher Scientific, Waltham, MA, USA) at around $240 \mathrm{mg} / \mathrm{mL}$, and then tightly encapsulated in aluminium hermetic pans. An empty pan was used as reference. They were scanned under dry nitrogen purge $(50 \mathrm{~mL} / \mathrm{min})$ from $-35{ }^{\circ} \mathrm{C}$ to $90{ }^{\circ} \mathrm{C}$, at a heating rate of 1 ${ }^{\circ} \mathrm{C} / \mathrm{min}$. Endothermic peak temperatures $\left(\mathrm{T}\right.$ peak, $\left.{ }^{\circ} \mathrm{C}\right)$ and enthalpies of conformational changes $(\Delta \mathrm{H}, \mathrm{J} / \mathrm{g})$ were determined at least in triplicate. TA Instruments Universal Analysis 2000 software was used for data analysis.

\subsubsection{Cryo-transmission electron microscopy}

234 Grids were prepared using freshly glow-discharged Quantifoil R2/2 holey carbon grids employing a Gatan cryo-plunge. For vitrification we used $3 \mu \mathrm{L}$ of sample per grid, 8-10 s of blotting time and liquid ethane. Specimen observation was carried out in a JEOL JEM-1230 transmission electron microscope operating at $100 \mathrm{kV}$ with a nominal magnification of $30 \mathrm{~K}$, using a Gatan 626 cryo-specimen holder. All micrographs were recorded at a temperature of about $-180{ }^{\circ} \mathrm{C}$ and under low-dose condition with a $4 \mathrm{k} \times 4 \mathrm{k}$ TVIPS CMOS camera detector (TemCam-F416).

\subsection{Statistical analysis}

The SPSS ${ }^{\circledR}$ computer program (IBM SPSS Statistics 22 Software, Inc., Chicago, IL, USA) was used to perform one-way analysis of variance. Differences between pairs of means were assessed using the Tukey test, with a significance level set at $p \leq 0.05$. 


\subsubsection{Chemical composition}

The results of proximate composition, tocopherol and residual amino acid contents of the commercial soybean lecithin (SL), as well as the two-step and five-step acetone-washed partially purified phosphatidylcholines (PW2 and PW5, respectively), are presented in Table 3. No significant differences $(p>0.05)$ in main constituents were found between the two washed PW samples. Crude lipid percent decreased significantly $(p \leq 0.05)$ in both PW2 and PW5 with respect to the SL sample, suggesting preferential removal of neutral lipids in the washed samples. The relatively low percentage values of crude lipids found in the three raw materials could be due to not taking into account a certain amount of polar lipids in the non-organic solvent phase which was discarded. Moreover, some sterol glycosides, free carbohydrates and sugars bound to lipid constituents might have been concentrated in the acetone-insoluble fraction, thus contributing to a further decrease in the relative amount of crude lipids in the washed samples. The increase in the total ash percentage found in the two PW samples was attributed mainly to the relative concentration of the phosphate groups of phospholipids. Moisture level was rather low in all cases (around 6\%), with no significant differences ( $p>0.05$ ) among samples. Similar trace amounts of protein were detected in SL, PW2 and PW5 (around $0.28 \%)$. The amino acid composition of the protein fraction in SL revealed the predominance of glutamic acid and aspartic acid, together with noticeable amounts of serine, lysine and arginine, among others. All amino acids decreased significantly $(p \leq 0.05)$ in both PW2 and PW5, with the exception of cysteine residues, which did not change significantly, and phenylalanine, which increased considerably $(p \leq 0.05) n$ both PW samples, probably because of its stronger lipophilic nature. 
preparations, $\boldsymbol{y}$-tocopherol was the most abundant, followed by $\delta$ - and $\alpha$-tocopherol. The

273 number of acetone washing steps induced significant differences $(p \leq 0.05)$ in the total contents of the three tocopherol species. The results for tocopherol in the present work are noticeably higher than those reported previously in another commercial soy lecithin, in which a total of 96\% reduction by acetone washing was found (Wang \& Wang, 2008). Tocopherol concentrations ranging from 48.9 to $188.6 \mathrm{mg} / 100 \mathrm{~g}$ in soy oil have been shown to depend on the extraction and analytical methodologies used (Méjean, Brunelle \& Touboul, 2015). Acetone has been reported to exert a powerful solubilizing effect on tocopherol (Wayno, Kaewseejan \& Siriamornpun, 2016), contributing greatly to efficient removal of tocopherol in the process of phospholipid concentration. However, when considering the use of partially purified phosphatidylcholine for production of food grade liposomes, the residual presence of tocopherol in PW2 could provide interesting protection of the unsaturated fatty acid of lipid membrane against oxidation (Carvajal, Rustad, Mozuraityte \& Storrø, 2009).

The results of fractionation of SL, PW2 and PW5 samples into the various classes of lipids is shown in Figure 1. Phospholipids were the most abundant fraction in the soy lecithin (57.55\%), followed by neutral lipids (38.34\%) and free fatty acids (4.11\%). The acetone washing steps led to a similar $(p>0.05)$ reduction $(\approx 90 \%)$ of neutral lipids in both PW2 and PW5. Despite being the least abundant fraction, the removal of free fatty acids was significantly higher $(p \leq 0.05)$ in PW5 than in PW2 (78\% vs. 71\%). The percentage of phospholipids was markedly increased in both PW samples, representing around 95\% of lipids, in agreement with Van Hoogevest and Wendel (2014). No significant differences $(p>0.05)$ in the total amount of phospholipids were found depending on the number of washing steps, which indicates that the two acetone washings in PW2 were mostly enough for extracting the phospholipid fraction. For use in the food industry, this level of phosphatidylcholine purification would probably be enough for most applications, with the advantage of having potential antioxidant capacity due to the remaining $\alpha$-tocopherol content. 
Table 4 presents the fatty acid composition of the three main lipid fractions (neutral lipids, free

299 fatty acids and phospholipids) of the SL, PW2 and PW5 samples. The most abundant fatty acids in SL were linoleic (C18:2n6c), oleic (C18:1n9c), palmitic (C16:0), linolenic (C18:3n3) and stearic acid (C18:0), all of them being present in the three lipid fractions studied. No significant differences $(p>0.05)$ in the fatty acid profile were observed in the phospholipid fraction of SL in comparison to the two PW samples. Similar fatty acid composition in commercial soy lecithin and acetone-washed phosphatidylcholine has been reported previously (Van Nieuwenhuyzen \& Tomas, 2008; Wang \& Wang, 2008; Lee, Weng \& Su, 2015). However, a sharp decrease in the content of all fatty acids upon two washing steps was found in neutral lipids and free fatty acids. Five washing steps in PW5 led to a further $(p \leq 0.05)$ reduction in most cases as compared total of 41 phospholipid species were identified in the three tested samples, none of which having two saturated fatty acids in the same molecule (Table 1). Phosphatidylserine was not 

$(p \leq 0.05)$ concentration being found in the more washed sample (PW5), which was 1.3-fold concentrated with respect to the parent SL. Noticeable amounts of phosphatidylethanolamine (PE), as well as lyso forms of PC and PE, were also detected in the positive mode, with a similar ( $p>0.05)$ degree of washing-induced concentration in all cases. Phosphatidylinositol (PI) and phosphatidic acid (PA) were only detected in the negative mode (Figure 2b). Both of them were around 2.1 times more concentrated in washed samples than in the parent SL, with no differences $(p>0.05)$ regarding the number of washing steps. Relatively high levels of anionic phospholipid classes, such as PI and PA, have also been reported, along with the more abundant PC and PE, in soy lecithin (Sikorski \& Kolakowska, 2003). In the present work, all phospholipid classes were considerably concentrated in the first two-step washing of lecithin. Further washing in the PW5 sample led to a significant increase $(p \leq 0.05)$ only in the concentration of PC, as compared to the less washed PW2. It should be noted that absolute comparisons regarding the concentrations of the various phospholipid classes determined in both positive and negative ionization modes were not possible; however, for instance, the maximum signal intensity registered in the positive mode for PC in the PW5 sample was 164 times greater than the intensity measured in the negative mode for PI. This finding strongly suggested a clear predominance of PC over all other classes of phospholipids present in the washed PW samples, providing evidence that the use of acetone is an excellent method for isolating phosphatidylcholine.

Besides the considerable removal of non-phospholipid components in both PW samples as compared to SL, slight differences were found between the two acetone-washed samples depending on the number of washing steps. Thus, the more intense acetone washing procedure in PW5 as compared to PW2 contributed mainly to increasing the concentration of 
acids) and the residual tocopherol content. Nevertheless, a certain amount of protein and

350 undetermined residual material remained in both acetone-insoluble fractions.

\subsubsection{Infrared spectroscopy (ATR-FTIR)}

352

Partial phospholipid purification induced by acetone washing was also evidenced by FTIR. The IR spectra of soy lecithin (SL) and partially purified phosphatidylcholines after two-step and five-step acetone washing (PW2 and PW5, respectively) are shown in Figure 3. The IR absorption band at $\approx 3270 \mathrm{~cm}^{-1}$, corresponding to the stretching vibration of $\mathrm{O}-\mathrm{H}$ and $\mathrm{N}-\mathrm{H}$ groups of water molecules, alcohols and/or amines, showed a notable increase in intensity and amplitude in both PW samples as compared to SL. This increase could not be attributed to higher moisture or tocopherol contents, since no differences in moisture level were found among the three samples, and a considerable amount of tocopherol was removed by successive washing. The higher absorption could therefore be attributed to concentration of the amino groups of the phospholipid head groups (mainly choline and also ethanolamine) in the two PW samples. The higher intensity in PW5 coincided with a slightly higher purification level.

The increase in phospholipid concentration was also evidenced by changes in the intensity of other IR bands. The slight reduction in the band at $\approx 1740 \mathrm{~cm}^{-1}$, assigned to the stretching vibration of the ester $\mathrm{C}=\mathrm{O}$ groups, corresponded mainly to the removal of triglycerides in the two PW samples. The higher intensities at $\approx 1655$, assignable to $C=C$ stretching vibration of cisolefins, at $\approx 1230 \mathrm{~cm}^{-1}$, representing the $\mathrm{PO}_{2}^{-}$antisymmetric double bond stretching, and at $\approx 1050 \mathrm{~cm}^{-1}$, mainly assigned to $\mathrm{C}-\mathrm{O}$ groups of glycerol and $\mathrm{C}-\mathrm{N}$ groups of choline, were also indicative of noticeable phospholipid concentration.

Interestingly, the peak shift from 1742 in SL to $1737 \mathrm{~cm}^{-1}$ in the two PW samples, along with the peak intensity decrease, could also be indicative of the possible appearance of aldehydes and ketones, which are secondary oxidation products whose absorbance is near $1728 \mathrm{~cm}^{-1}$ 
374 (Guillén \& Cabo, 2004). The presence of thiobarbituric acid reactive substances (TBARS)

375 induced by lipid oxidation has been reported to occur in soy lecithin emulsions, their 376 accumulation being influenced not only by the fatty acid profile but also by the PL class 377 composition and net electric charge (Wang \& Wang, 2008).

\subsection{Liposome characterization}

In order to know if the slight compositional differences found in both acetone-washed materials may affect liposomal properties, PW2 and PW5, as well as the parent SL, were used to produce liposomes by applying two methods of sonication (see Table 2).

\subsubsection{Size, polydispersity and zeta potential}

All fresh liposomal dispersions presented typical monomodal particle size distributions with zaverage in the range of 74 to $132 \mathrm{~nm}$ (Figure 4; Table 5), in agreement with previous work using the sonication procedure for liposome production (Drazenovic et al., 2015; Klingler, Vargas, Fiedler \& Keller, 2015). The polydispersity index varied among samples, ranging from 0.17 in L2A to 0.27 in L5Ang (Table 5). These values are similar to the range between 0.2 and 0.3 expected for systems prepared from biological materials (Da Silva Malheiros, et al., 2010).

Comparison of all the glycerol-containing liposomes showed that the LSL dispersion had the highest particle size and L5A had the lowest one. Thus, the more intense washing in PW5, together with the stronger sonication procedure, contributed noticeably to reducing the vesicle size. A linear decrease in particle size and polydispersity of unilamellar liposomes as a result of increasing the time and intensity of sonication has been reported previously (Silva et al., 2010). Regardless of which sonication procedure was used, the larger vesicle size of the LSL liposomes could be attributed to the presence of non-phospholipid compounds, mainly triglycerides and tocopherol that could be trapped inside the bilayer (Michelon, Mantovani, 
effect could be ascribed to the presence of glycerol, since the liposomal preparation without glycerol (L5Ang) presented a significantly lower particle size than the glycerol-containing counterpart (L5A). This result was in agreement with Manca et al. (2013), who reported a significant increase in particle size when using glycerol concentrations above $20 \%$.

The zeta potential was strongly electronegative in all the liposomal dispersions, indicative of high particle stability (Müller, Jacobs \& Kayser, 2001) (Table 5). Negative membrane surface charge could be explained on the basis of the PL class composition of the liposomes. At neutral $\mathrm{pH}$, the negative charge of phosphorus groups is normally counteracted by the positive charge of choline and ethanolamine groups, resulting in the absence of net charge in PC and PE. However, the relatively high content of PI found in the three materials studied would result in a negative net charge (Wang \& Wang, 2008). The presence of other surface-active impurities, such as free fatty acids and protein traces, might also have contributed to the electronegative zeta potential of the liposomal dispersions (McClements, 2016). In contrast, little effect was attributed to the remaining relatively high tocopherol content in the materials studied, in agreement with Fukuzawa et al. (1992), who found poor exposure of $\alpha$-tocopherol at the surface of a model phospholipid membrane. A clear relationship between zeta potential and purification level or sonication intensity was not found, but less electronegative potentials were observed in the smallest liposomes made from the most purified phosphatidylcholine material using the stronger sonication, irrespective of the presence of glycerol (L5A and L5Ang). Therefore the use of glycerol seemed not to be a critical factor in the liposomal surface charge, as observed before in phospholipid vesicles made with high glycerol concentrations (Manca et al., 2013).

Purification level and sonication intensity parameters separately did not induce significant changes in particle size and zeta potential, probably because the differences in the two washed samples (PW2 and PW5) were not very pronounced. Silva et al. (2010) also found no 
significant differences in zeta potential regarding the time and intensity of sonication. However, the liposomal dispersion with the highest purification and the stronger sonication was clearly distinguished from the others, both in particle size and in zeta potential, regardless of the presence of glycerol. The slightly higher phosphatidylcholine concentration and lower residual non-phospholipid impurities (tocopherol, aminoacids, free fatty acids), as well as conformational changes induced by the more intense sonication procedure, might have jointly influenced the distinctive hydrodynamic and surface properties of L5A samples. However, for many food applications in which the particle size does not represent a limiting factor, liposomes obtained from the less washed PW2 (L2A or L2B) would represent a feasible alternative.

\subsubsection{Thermal properties}

Within the temperature range studied (from $-35^{\circ} \mathrm{C}$ to $90{ }^{\circ} \mathrm{C}$ ), all the concentrated liposomal dispersions showed a main cooperative endothermic transition at subzero temperatures $\left(T_{\text {peak } 1}\right)$, ranging from -1.7 to $-8.9{ }^{\circ} \mathrm{C}$ (Figure 5 ; Table 6 ). The negative range is typical for liposomes made of soy phosphatidylcholine with a high content of polyunsaturated fatty acids (Wang \& Briggs, 2002). In particular, the presence of even a single cis double bond in the acyl chain of the phospholipid molecule would be enough to alter the lipid packing within the bilayer, resulting in a very large decrease of Tm as compared to the highly saturated synthetic phosphatidylcholines, in which the main gel to liquid-crystalline transition takes place at around $41^{\circ} \mathrm{C}$ (Biltonen \& Lichtenberg, 1993). Comparison of liposomes produced by the same sonication procedure (method A) showed that the main $T_{\text {peak } 1}$ decreased progressively as the number of washing steps increased, from $-6.3^{\circ} \mathrm{C}$ in $L S L$ and $-7.8^{\circ} \mathrm{C}$ in $L 2 A$ to $-8.9^{\circ} \mathrm{C}$ in L5A. This effect coincided with an increase in the relative concentration of total phospholipids in acetone-washed samples, and therefore with an increase in the amount of linoleic acid

447 (C18:2n6c), since the amount of this fatty acid as compared to the others was highest in the 
phospholipid fraction (Table 4). On the other hand, using the same washed material (PW2 or PW5), liposomes made by strong sonication (method A) showed slightly lower $T_{\text {peak } 1}$ than

450 liposomes made by method B. This effect could be indicative of a higher prevalence of small unilamellar vesicles (SUVs) with the more intense sonication method (Taylor \& Morris, 1995). Among all the glycerol-containing liposomes, the change in enthalpy $(\Delta \mathrm{H})$ was significantly lower in liposomes produced from the most purified material using the stronger sonication method (L5A), probably as a result of less prevalence of large unilamellar vesicles (LUVs) in the vesicle population (Biltonen \& Lichtenberg, 1993). The increase in the concentration of phospholipids played a key role in reducing the change in enthalpy of the resulting liposomes only when the strong sonication procedure was used. However, under these conditions, liposomes without glycerol (L5Ang) showed the highest values of both $\mathrm{T}_{\text {peak } 1}\left(-1.7^{\circ} \mathrm{C}\right)$ and $\Delta \mathrm{H}$, regardless of the purification level or sonication method, strongly suggesting that glycerol interfered in the membrane structure.

Besides this main thermal event, another endothermic transition, $T_{\text {peak2 }}$, was also observed in all the liposomal dispersions at temperatures around $10^{\circ} \mathrm{C}$ (Figure 5, inset; Table 6). Comparison of the glycerol-added liposomes showed that the value of $T_{\text {peak2 }}$ was significantly $(\mathrm{p} \leq 0.05)$ higher in $\mathrm{L} 5 \mathrm{~A}\left(11.3^{\circ} \mathrm{C}\right)$ and lowest in L2B $\left(7.9^{\circ} \mathrm{C}\right)$, with intermediate temperatures in the other samples $\left(9.0-9.2^{\circ} \mathrm{C}\right)$. Regardless of the raw material, sonication method B slightly reduced both the $T_{\text {peak2 }}$ and the associated enthalpy change, which could be indicative of less stable packing of the bilayer. The L5A preparation (higher purification and stronger sonication) was again slightly distinguished from the others regarding this transition, the higher peak temperature and enthalpy change suggesting a more ordered membrane, with higher rigidity and stability (Christensen et al., 2007), in clear contrast to L2B (lower purification and weaker sonication). The liposomes without glycerol (L5Ang) showed a slightly different melting pattern, with two minor transitions $\left(\mathrm{T}_{\text {peak } 2}\right.$ at $8.4{ }^{\circ} \mathrm{C}, \Delta \mathrm{H}=2.20 \mathrm{~J} / \mathrm{g}$; and $\mathrm{T}_{\text {peak3 }}$ at $17 .{ }^{\circ} \mathrm{C}, \Delta \mathrm{H}=$ 
474 (Figure 5, inset). This difference could be attributed to the irruption of glycerol into the

475 membrane packing, causing increased hydration of the lipid bilayer (Manca et al., 2013). No more thermal events were found in any of the studied samples at temperatures up to $90^{\circ} \mathrm{C}$.

\subsubsection{Cryo-transmission electron microscopy}

The morphology of liposomes LSL, L5A and L5Ang, which were selected for having very different properties, was examined by Cryo-TEM (Figure 6). The LSL images showed a relatively heterogeneous morphology with a significant amount of large and non-spherical vesicles, typically attributed to the presence of impurities and a high degree of unsaturation of the acyl chains in soy lecithin (Rangelov, Momekova \& Almgren, 2010). In contrast, well-separated spherical unilamellar vesicles, with a smaller particle size, predominated in both L5A and L5Ang aqueous dispersions, in accordance with DLS results. In both preparations, in addition to small unilamellar vesicles, many bivesicular vesicles were observed, and in the case of liposomes without glycerol a number of flattened liposomes were also observable. It must be noted that liposomes without glycerol were more easily visualized, probably because they presented a different affinity for the holey carbon grid in the microscope.

\section{Conclusions}

491 It is well established that acetone is a solvent of choice for concentrating the fraction of 492 phospholipids present in soy lecithin. In the present work it has been shown that an increase in the number of washes with acetone contributes to a progressive reduction in the content of neutral lipids, free fatty acids and tocopherol; however, it does not modify the fatty acid profile of the phospholipid fraction, where linoleic acid (C18:2n6c) predominates, representing $59 \%$ of this fraction, both in lecithin and in partially purified phosphatidylcholines. With five washing steps a significant concentration of PC was achieved compared to only two washes, 

provided evidence of the concentration of phospholipids and also suggested possible accumulation of aldehydes and ketones in both washed materials. All liposomal dispersions presented very high particle stability, the washing with acetone contributing significantly to a decrease in the average particle size with respect to the parent lecithin. However, the effect of further washing on size became significant only when it coincided with an intense sonication process (L5A liposomes). For food application purposes, the purity of the soy phosphatidylcholine preparation achieved with the first two washing steps (PW2) would generally be enough to obtain derived liposomes with suitable structural properties. In addition, the higher residual amount of $\alpha$-tocopherol could probably provide better endogenous protection against oxidation. The production of liposomes with addition of

509 glycerol caused a slight interference in the membrane increasing the average particle size, but

510 it did not modify the membrane surface charge.

\section{Acknowledgements}

512 The authors wish to thank the Spanish Ministry of Economy and Competitiveness for financial support through project AGL2014-52825. We are grateful to the Analysis Service Unit facilities of ICTAN-CSIC for the Differential Scanning Calorimeter analysis (Rubén Domínguez) and the chromatographic analysis (Sonia Jiménez and Beatriz Díaz), as well as Electron Microscopy Facility (EMF) at the CIB-CSIC for CryoTEM analysis. 
Akbarzadeh, A., Rezaei-Sadabady, R., Davaran, S., Joo, S.W., Zarghami, N., Hanifehpour, Y., Samiei, M., Kouhi, M., Nejati-Koshki, K. (2013). Liposome: Classification, preparation, and applications. Nanoscale Research Letters, 8 (1), 1-8.

522 Álvarez, I., De La Fuente, J., Cañeque, V., Lauzurica, S., Pérez, C., Díaz, M.T. (2009). Changes in the fatty acid composition of $\mathrm{M}$. longissimus dorsi of lamb during storage in a high-oxygen modified atmosphere at different levels of dietary vitamin E supplementation. Journal of Agricultural and Food Chemistry, 57 (1), 140-146.

Alemán, A., Gómez-Guillén, M.C., \& Montero, P. (2013). Identification of ace-inhibitory peptides from squid skin collagen after in vitro gastrointestinal digestion. Food Research International, 54, 790-795. Guillén, M.C. (2016). A novel functional wrapping design by complexation of $\varepsilon$-polylysine with liposomes entrapping bioactive peptides. Food and Bioprocess Technology, 9, 1113-1124. Analytical Chemists. Biltonen, R.L., \& Lichtenberg, D. (1993). The use of differential scanning calorimetry as a tool to characterize liposome preparations. Chemistry and Physics of Lipids, 64 (1-3), 129-142. Bligh, E.G., \& Dyer, W.J. (1959). A rapid method for total lipid extraction and purification. Canadian Journal of Biochemistry and Physiology, 37, 911-917. cholesterol on drug encapsulation studies by phospholipid liposomes. Pharmaceutical Development and Technology, 16 (4), 408-414. 
541 Carvajal, A.K., Rustad, T., Mozuraityte, R., \& Storrø, I. (2009). Kinetic studies of lipid oxidation

542 induced by hemoglobin measured by consumption of dissolved oxygen in a liposome model system. Journal of Agricultural and Food Chemistry, 57 (17), 7826-7833.

544 Christensen, D., Foged, C., Rosenkrands, I., Nielsen, H.M., Andersen, \& P., Agger, E.M. (2007).

545 Trehalose preserves DDA/TDB liposomes and their adjuvant effect during freeze-drying.

546 Biochimica et Biophysica Acta-Biomembranes, 1768 (9), 2120-2129.

547 Da Silva Malheiros, P., Daroit, D.J., \& Brandelli, A. (2010). Food applications of liposome548 encapsulated antimicrobial peptides. Trends in Food Science and Technology, 21 (6), 284-292.

549 Drazenovic, J., Wang, H., Roth, K., Zhang, J., Ahmed, S., Chen, Y., Bothun, G., \& Wunder, S.L. 550 (2015). Effect of lamellarity and size on calorimetric phase transitions in single component 551 phosphatidylcholine vesicles. Biochimica et Biophysica Acta - Biomembranes, 1848 (2), 532552543.

553 Frenzel, M., \& Steffen-Heins, A. (2015). Impact of quercetin and fish oil encapsulation on bilayer membrane and oxidation stability of liposomes. Food Chemistry, 185, 48-57.

Fukuzawa, K., Ikebata, W., Shibata, A., Kumadaki, I., Sakanaka, T., \& Urano, S. (1992). Location 556 and dynamics of $\alpha$-tocopherol in model phospholipid membranes with different charges.

557 Chemistry and Physics of Lipids, 63 (1-2), 69-75.

558 Gómez-Estaca, J., Calvo, M.M., Álvarez-Acero, I., Montero, P., Gómez-Guillén, M.C. (2017). 559 Characterization and storage stability of astaxanthin esters, fatty acid profile and $\alpha$-tocopherol 560 of lipid extract from shrimp (L. vannamei) waste with potential applications as food ingredient. 561 Food Chemistry, 216, 37-44. 
562 Guillén, M.D., \& Cabo, N. (2004). Study of the effects of smoke flavourings on the oxidative

563 stability of the lipids of pork adipose tissue by means of Fourier transform infrared 564 spectroscopy. Meat Science, 66 (3), 647-657.

565 Klingler, J., Vargas, C., Fiedler, S., \& Keller, S. (2015). Preparation of ready-to-use small 566 unilamellar phospholipid vesicles by ultrasonication with a beaker resonator. Analytical 567 Biochemistry, 477, 10-12.

568 Laye, C., McClements, D.J., \& Weiss, J. (2008). Formation of biopolymer-coated liposomes by 569 electrostatic deposition of chitosan. Journal of Food Science, 73 (5), N7-N15.

570 Lee, W.-J., Weng, S.-H., \& Su, N.-W. (2015). Individual Phosphatidylcholine Species Analysis by RP-HPLC-ELSD for Determination of Polyenylphosphatidylcholine in Lecithins. Journal of Agricultural and Food Chemistry, 63 (15), 3851-3858.

573 Li, J., Wang, X., Zhang, T., Wang, C., Huang, Z., Luo, Xi., \& Deng, Y. (2015). A review on 574 phospholipids and their main applications in drug delivery systems. Asian Journal of 575 Pharmaceutical Sciences, 10, 81-98.

576 Manca, M.L., Zaru, M., Manconi, M., Lai, F., Valenti, D., Sinico, C., \& Fadda, A.M. (2013). 577 Glycerosomes: A new tool for effective dermal and transdermal drug delivery. International 578 Journal of Pharmaceutics, 455, 66-74.

579 McClements, D. J. (2016). Interfacial properties and their characterization. In Food Emulsions: 580 Principles, Practices, and Techniques, 3rd Edition. CRC Press. Taylor \& Francis Group. Boca 581 Raton, FL, pp. 185-244.

582 Méjean, M., Brunelle, A., \& Touboul, D. (2015). Quantification of tocopherols and tocotrienols 583 in soybean oil by supercritical-fluid chromatography coupled to high-resolution mass spectrometry. Analytical and Bioanalytical Chemistry, 407 (17), 5133-5142. 
585

586

587

588

589

590

591

592

593

594

595

596

597

598

599

600

601

602

603

604

605

606

Michelon, M., Mantovani, R.A., Sinigaglia-Coimbra, R., de la Torre, L.G., \& Cunha, R.L. (2016).

Structural characterization of $\beta$-carotene-incorporated nanovesicles produced with nonpurified phospholipids. Food Research International, 79, 95-105.

Mosquera, M., Giménez, B., Mallmann da Silva, I., Ferreira Boelter, J., Montero, P., GómezGuillén, M.C., \& Brandelli, A. (2014). Nanoencapsulation of an active peptidic fraction from sea bream scales collagen. Food Chemistry, 156, 144-150.

Mozafari, M.R., Johnson, C., Hatziantoniou, S., \& Demetzos, C. (2008). Nanoliposomes and their applications in food nanotechnology. Journal of Liposome Research, 18 (4), 309-327.

Müller, R.H., Jacobs, C. \& Kayser, O. (2001). Nanosuspensions as particulate drug formulations in therapy: rationale for development and what we can expect for the future. Advanced Drug Delivery Reviews, 47 (1), 3-19.

Rangelov, S., Momekova D. \& Almgren M. (2010). Structural characterization of lipid-based colloidal dispersions using cryogenic transmission electron microscopy. In Microscopy: Science, Technology, Applications and Education. A. Méndez-Vilas \& J. Díaz (Eds.) FORMATEX Microscopy Series No. 4, VOL. 3., Badajoz, Spain, pp. 1724-1734.

Sikorski, Z.E.; \& Kolakowska, A. (2003). Chemical and Functional Properties of Food Lipids. New York: CRC Press.

Silva, R., Ferreira, H., Little, C., \& Cavaco-Paulo, A. (2010). Effect of ultrasound parameters for unilamellar liposome preparation. Ultrasonics Sonochemistry, 17 (3), 628-632.

Stark, B., Pabst, G. \& Prassl, R. (2010). Long-term stability of sterically stabilized liposomes by freezing and freeze-drying: Effects of cryoprotectants on structure. European Journal of Pharmaceutical Sciences, 41 (3-4), 546-555. 
607 Taylor, K.M.G., \& Morris, R.M. (1995). Thermal analysis of phase transition behaviour in 608 liposomes. Thermochimica Acta, 248 (C), 289-301.

609 Toyran, N., \& Severcan, F. (2003). Competitive effect of vitamin D2 and Ca2+ on phospholipid 610 model membranes: An FTIR study. Chemistry and Physics of Lipids, 123 (2), 165-176.

611 Van Hoogevest, \& P., Wendel, A. (2014). The use of natural and synthetic phospholipids as 612 pharmaceutical excipients. European Journal of Lipid Science and Technology, 116 (9), $1088-$ 6131107.

614 Van Nieuwenhuyzen, W., \& Tomás, M.C. (2008). Update on vegetable lecithin and 615 phospholipid technologies. European Journal of Lipid Science and Technology, 110 (5), 472616486.

617 Wang, G., \& Wang, T. (2008). Oxidative stability of egg and soy lecithin as affected by transition 618 metal ions and $\mathrm{pH}$ in emulsion. Journal of Agricultural and Food Chemistry, 56 (23), 1142461911431.

620 Wang, T., \& Briggs, J.L. (2002). Rheological and thermal properties of soybean oils with 621 modified FA compositions. Journal of the American Oil Chemists' Society, 79 (8), 831-836.

622 Wayno, P., Kaewseejan, N., \& Siriamornpun, S. (2016). Bioactive compounds and antioxidant 623 properties of different solvent extracts derived from Thai rice by-products. Applied Biological 624 Chemistry, 59(3), 373-384.

625 Yan, X., Li, H., Xu, J. \& Zhou, C. (2010) Analysis of phospholipids in microalga Nitzschia 626 closterium by UPLC-Q-TOF-MS. Chinese Journal of Oceanology and Limnology, 28: 106-112. 
629 FIGURE 1. Percent of main lipid fractions (Neutral lipids, Free fatty acids, and Phospholipids) 630 determined in soy lecithin (SL) and partially purified phosphatidylcholine after two acetone 631 washing steps (PW2) and five acetone washing steps (PW5). Different letters $(a, b, c)$ indicate 632 significant differences $(P \leq 0.05)$ as a function of washing steps.

633 FIGURE 2. Phospholipid class composition, expressed as signal intensity in positive (a) and 634 negative (b) polarity, of soy lecithin (SL) and partially purified phosphatidylcholine after two 635 acetone washing steps (PW2) and five acetone washing steps (PW5). PC: phosphatidylcholine; 636 PE: phosphatidylethanolamine; LPC: lysophosphatidylcholine; LPE: lysophosphatidyl637 ethanolamine; PI: phosphatidylinositol; PA: phosphatidic acid.

638 FIGURE 3. Infrared spectra (ATR-FTIR) of soy lecithin (SL) and partially purified 639 phosphatidylcholine after two acetone washing steps (PW2) and five acetone washing steps 640 (PW5).

641 FIGURE 4. Particle size distribution of soy lecithin liposomes (LSL), liposomes made of partially 642 purified phosphatidylcholine after two acetone washing steps ( $L 2 A$ and $L 2 B$ ) and five acetone 643 washing steps (L5A and L5B), and liposomes without glycerol made of partially purified 644 phosphatidylcholine after five acetone washing steps (L5Ang).

645 FIGURE 5. Differential Scanning Calorimetry (DSC) traces of soy lecithin liposomes (LSL), 646 liposomes made of partially purified phosphatidylcholine after two acetone washing steps (L2A 647 and L2B) and five acetone washing steps (L5A and L5B), and liposomes without glycerol made 648 of partially purified phosphatidylcholine after five acetone washing steps (L5Ang).

649 FIGURE 6. Cryo-TEM images of soy lecithin liposomes (LSL) and liposomes made of partially 650 purified phosphatidylcholine after five acetone washing steps with glycerol (L5A) and without 651 glycerol (L5Ang) produced by sonication method A. (1) spherical unilamellar vesicle, (2) 652 bivesicular liposome; (3) multivesicular liposome; (4) flattened liposome; (5) bilamellar 653 liposome; (6) non-spherical liposome. 


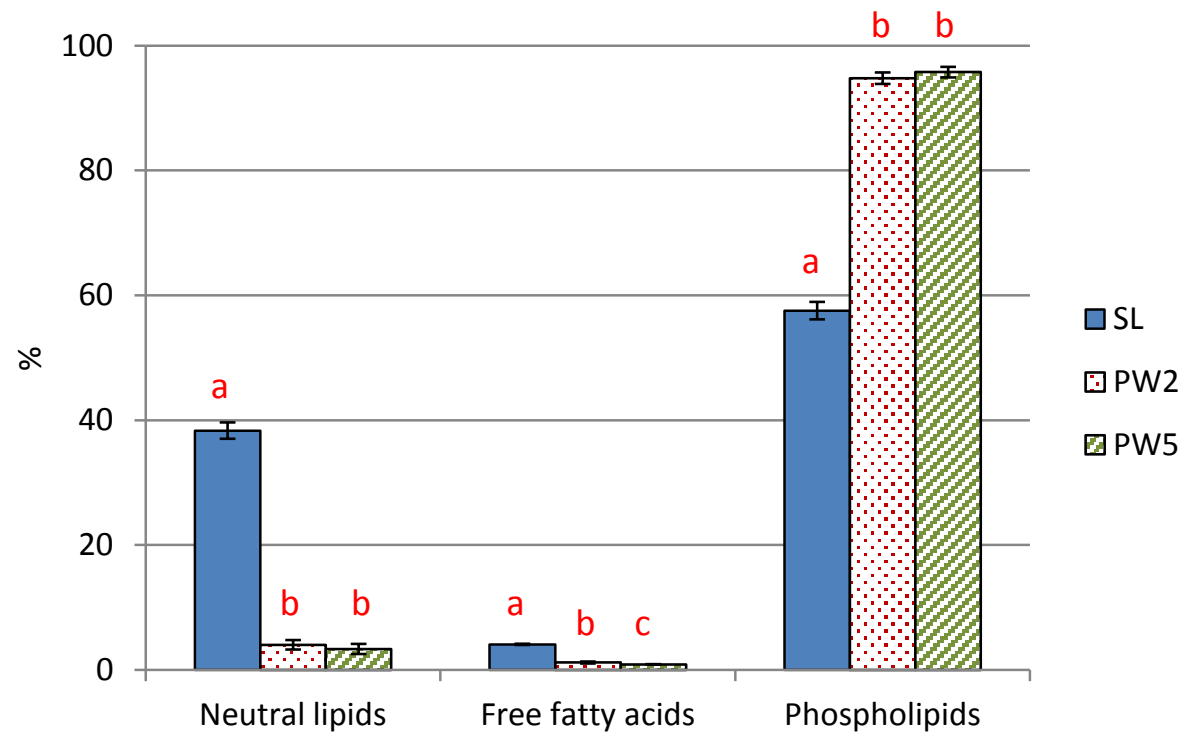

Figure 1. 


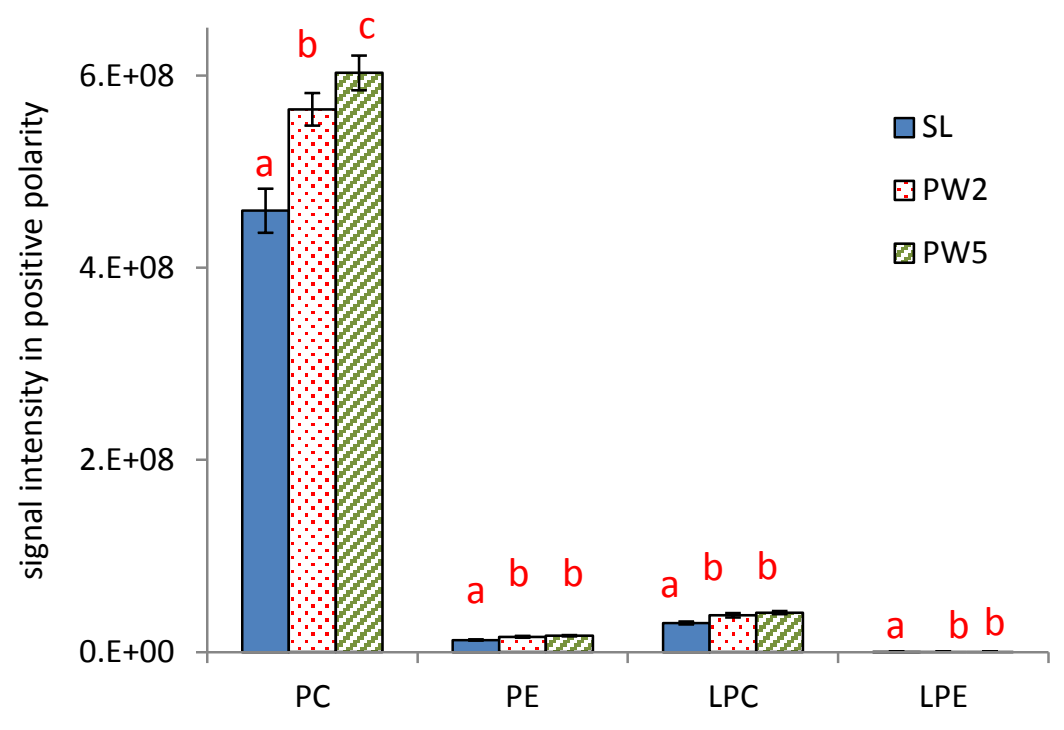

(a)

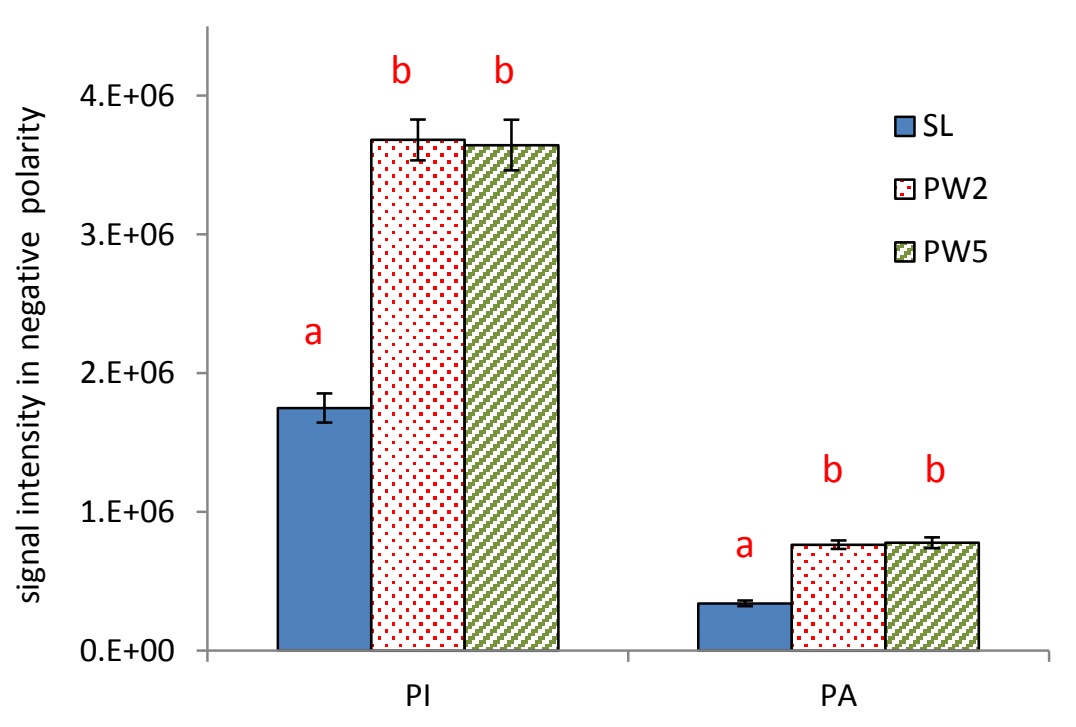

(b)

Figure 2 
Figure 3

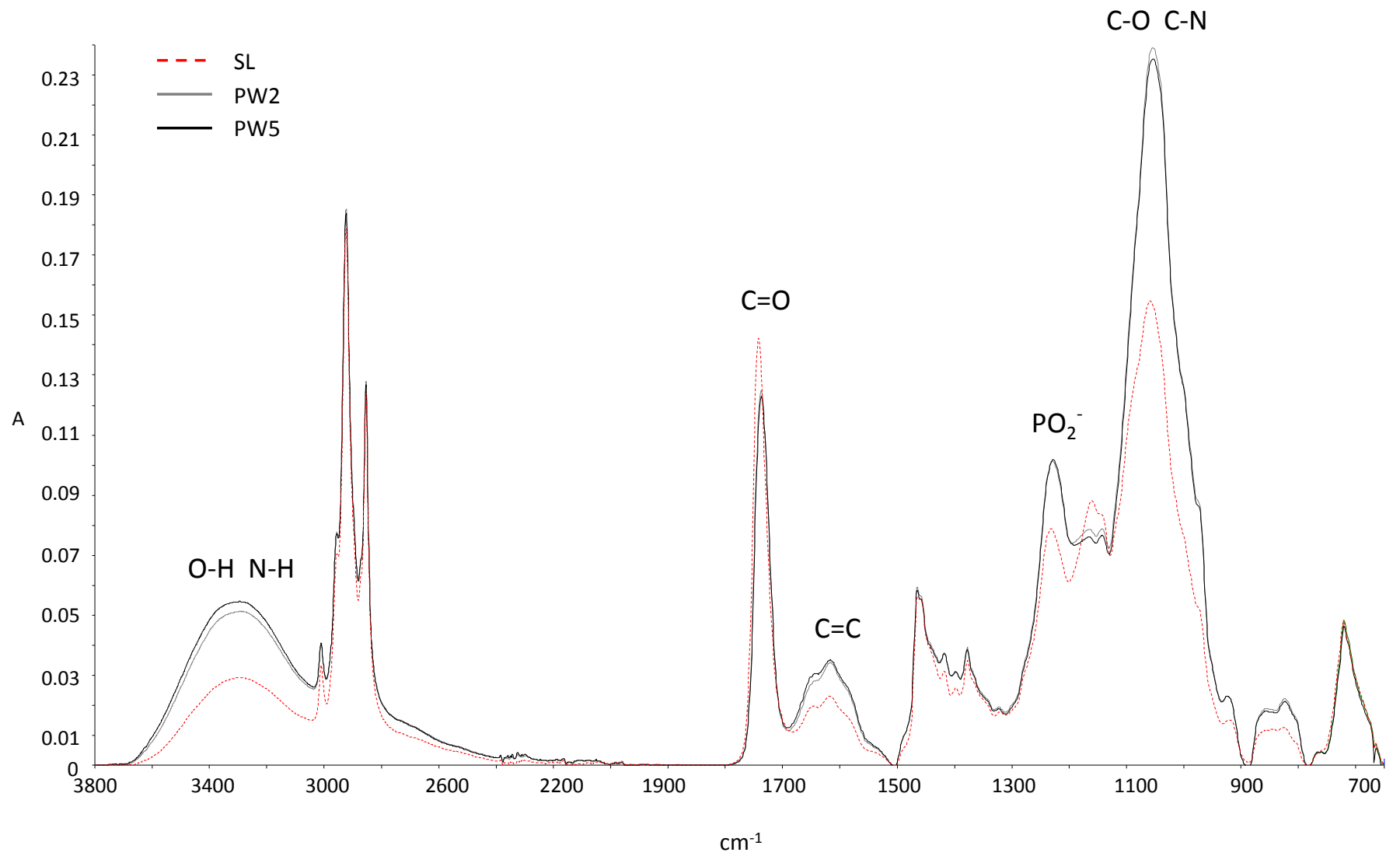

Figure 3 


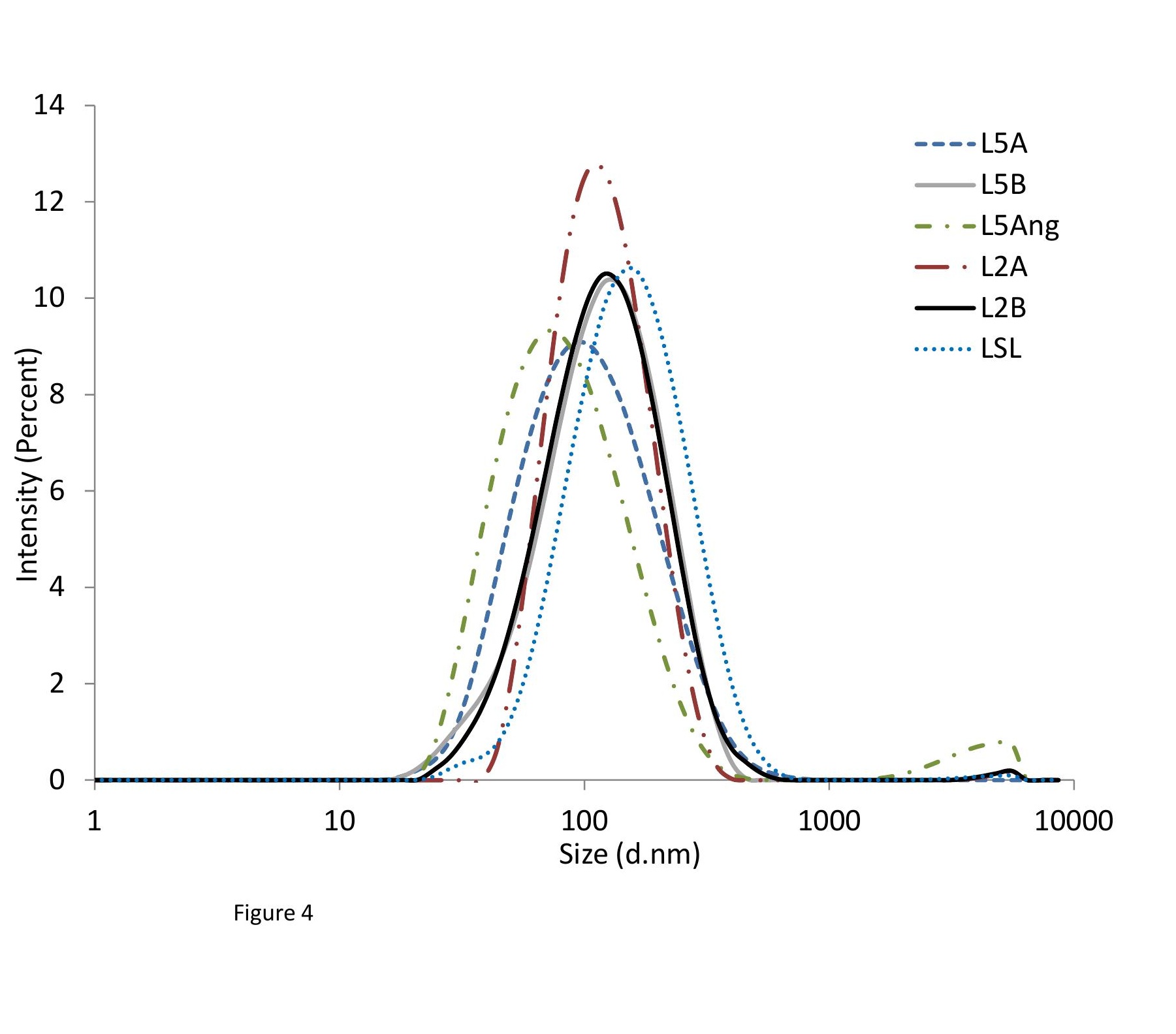

Figure 4

Figure 4

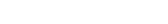

Figure 4

\section{Figure 4}

(1)
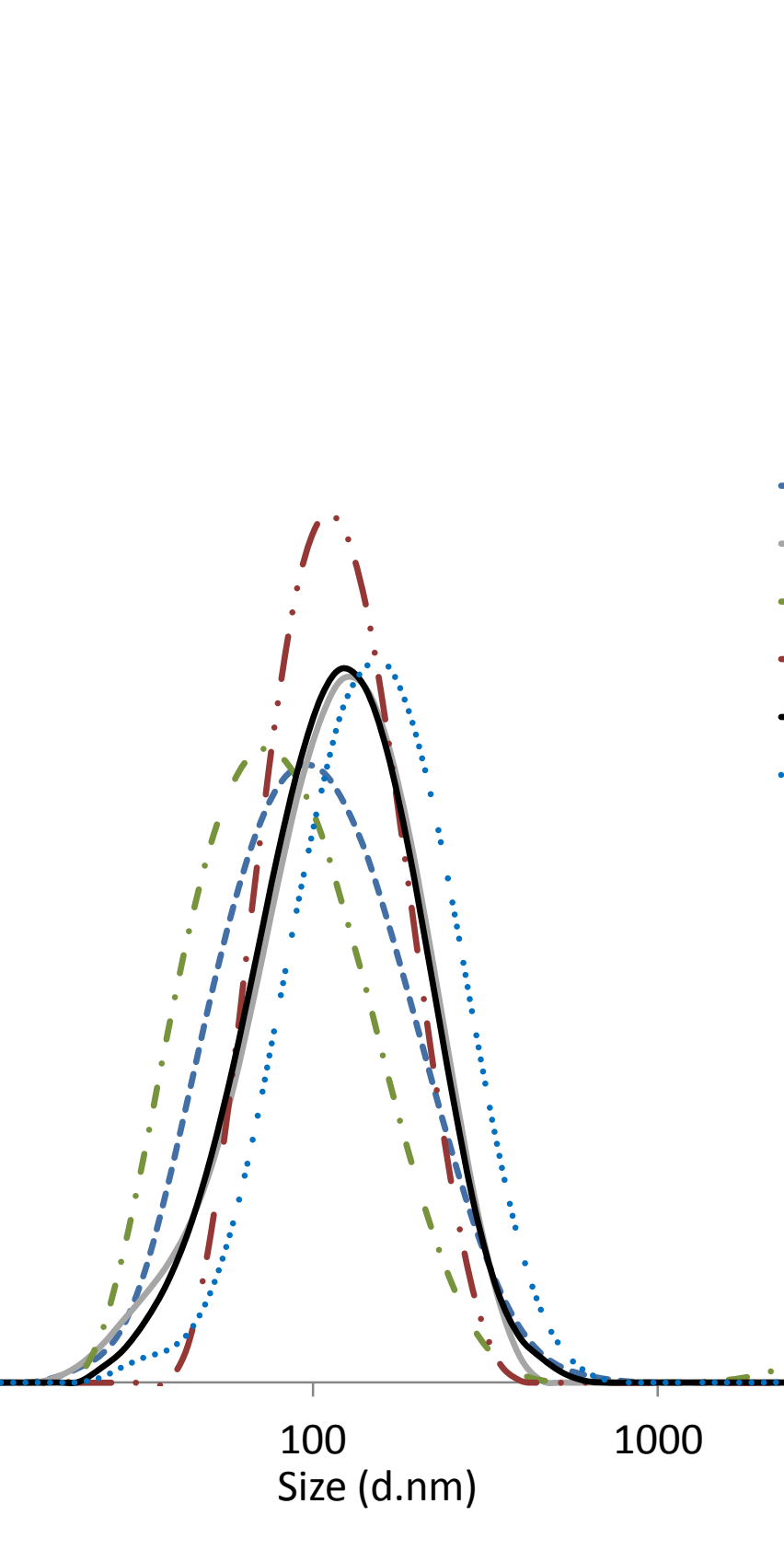

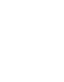

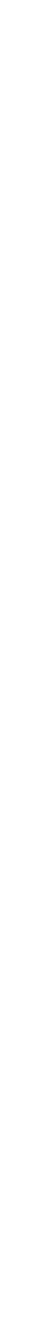

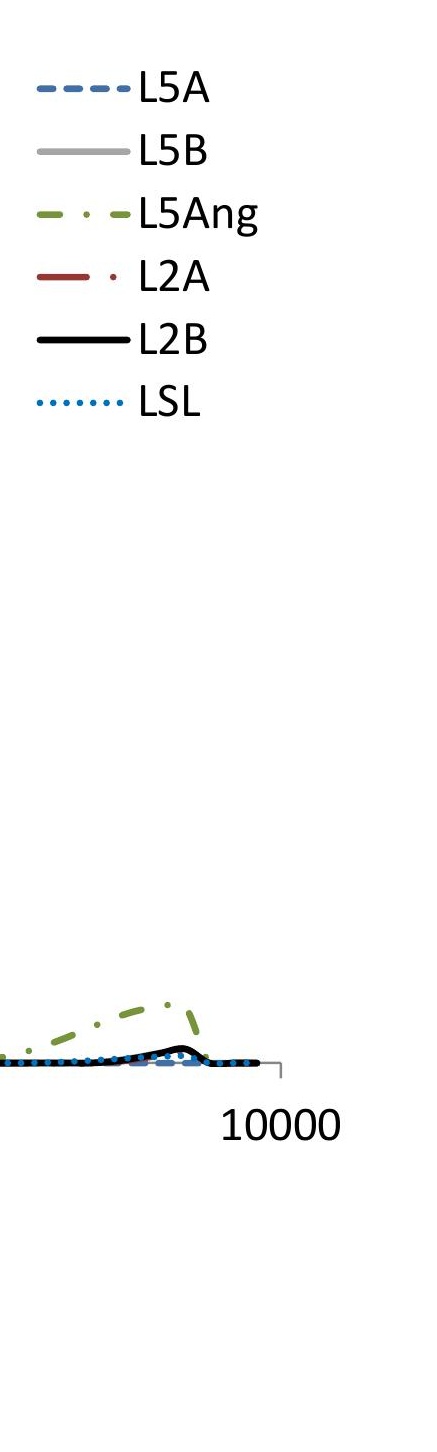


Figure 5

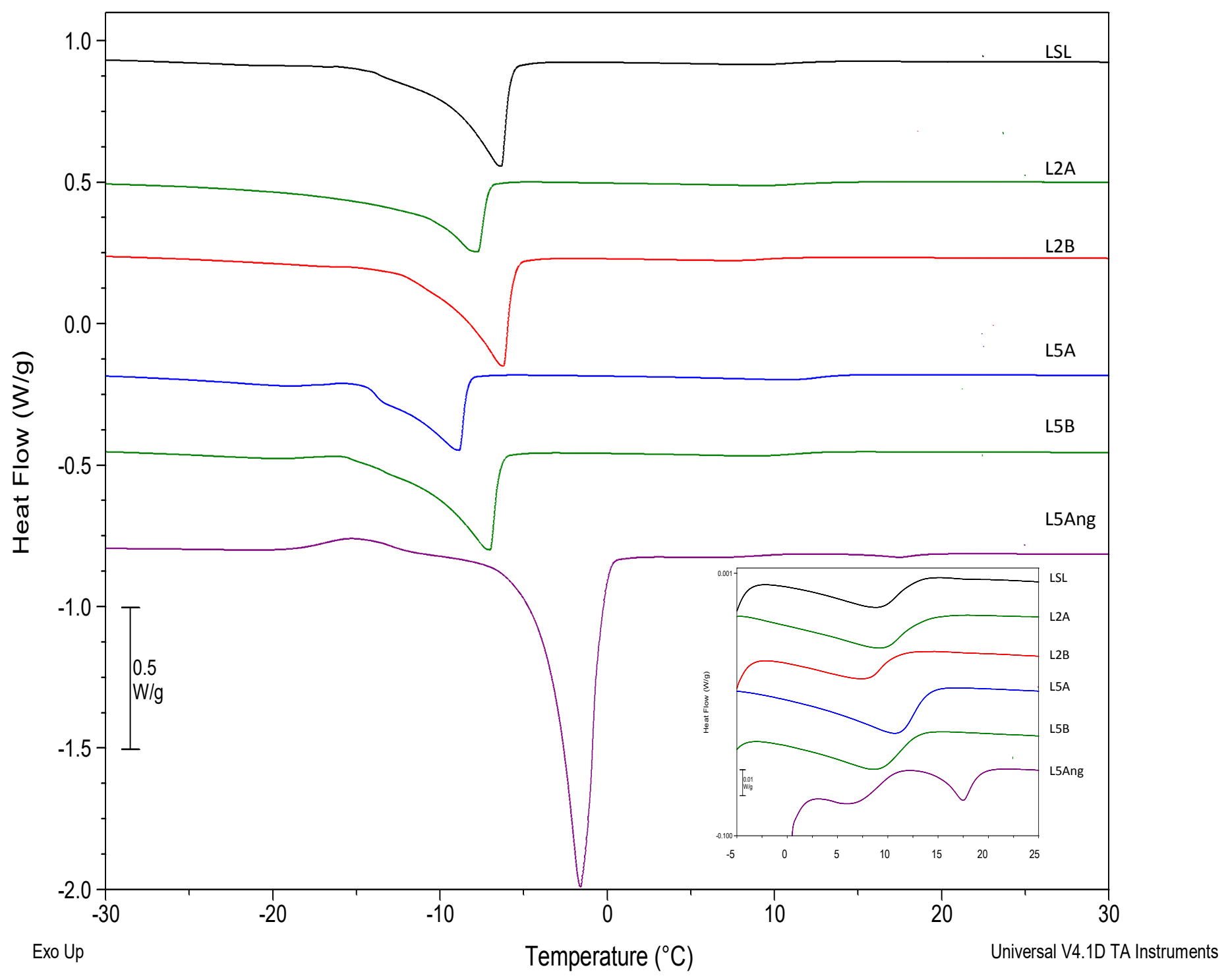

Figure 5 


$$
\text { I }
$$


Table 1.- Identification of different phospholipid species in tested samples.

\begin{tabular}{|c|c|c|}
\hline Polar head & Fatty acid combination & Match formula \\
\hline Choline & C16:0/C18:3n3 & $\mathrm{C} 42 \mathrm{H} 7808 \mathrm{NP}$ \\
\hline Choline & $\mathrm{C} 16: 0 / \mathrm{C} 18: 2 \mathrm{n} 6 \mathrm{c}$ & $\mathrm{C} 42 \mathrm{H} 8008 \mathrm{NP}$ \\
\hline Choline & $\mathrm{C} 16: 0 / \mathrm{C} 18: 1 \mathrm{n} 9 \mathrm{c}$ & $\mathrm{C} 42 \mathrm{H} 82 \mathrm{O} 8 \mathrm{NP}$ \\
\hline Choline & C18:3n3/C18:3n3 & $\mathrm{C} 44 \mathrm{H} 7608 \mathrm{NP}$ \\
\hline Choline & $\mathrm{C} 18: 2 \mathrm{n} 6 \mathrm{c} / \mathrm{C} 18: 3 \mathrm{n} 3$ & $\mathrm{C} 44 \mathrm{H} 7808 \mathrm{NP}$ \\
\hline Choline & $\mathrm{C} 18: 2 \mathrm{n} 6 \mathrm{c} / \mathrm{C} 18 \mathrm{a} 2 \mathrm{n} 6 \mathrm{c}$ & $\mathrm{C} 44 \mathrm{H} 8008 \mathrm{NP}$ \\
\hline Choline & $\mathrm{C} 18: 3 n 3 / C 18: 1 n 9 c$ & C44H8008NP \\
\hline Choline & C18:0/C18:3n3 & $\mathrm{C} 44 \mathrm{H} 82 \mathrm{O} 8 \mathrm{NP}$ \\
\hline Choline & $\mathrm{C} 18: 2 \mathrm{n} 6 \mathrm{c} / \mathrm{C} 18 \mathrm{in} 9 \mathrm{c}$ & $\mathrm{C} 44 \mathrm{H} 82 \mathrm{O} 8 \mathrm{NP}$ \\
\hline Choline & $\mathrm{C} 18: 0 / \mathrm{C} 18: 2 \mathrm{n} 6 \mathrm{c}$ & $\mathrm{C} 44 \mathrm{H} 8408 \mathrm{NP}$ \\
\hline Choline & C18:1n9c/C18:1n9c & C44H84O8NP \\
\hline Choline & C18:0/C18:1n9c & C44H86O8NP \\
\hline Ethanolamine & C16:0/C18:3n3 & C39H7108NP \\
\hline Ethanolamine & $\mathrm{C} 16: 0 / \mathrm{C} 18: 2 \mathrm{n} 6 \mathrm{c}$ & $\mathrm{C} 39 \mathrm{H} 7308 \mathrm{NP}$ \\
\hline Ethanolamine & $\mathrm{C} 16: 0 / \mathrm{C} 18: 1 \mathrm{n} 9 \mathrm{c}$ & $\mathrm{C} 39 \mathrm{H} 7508 \mathrm{NP}$ \\
\hline Ethanolamine & $\mathrm{C} 18: 2 \mathrm{n} 6 \mathrm{c} / \mathrm{C} 18: 3 \mathrm{n} 3$ & $\mathrm{C} 41 \mathrm{H} 7108 \mathrm{NP}$ \\
\hline Ethanolamine & $\mathrm{C} 18: 2 \mathrm{n} 6 \mathrm{c} / \mathrm{C} 18 \mathrm{2n} 6 \mathrm{c}$ & C41H7308NP \\
\hline Ethanolamine & $\mathrm{C} 18: 3 \mathrm{n} 3 / \mathrm{C} 18: 1 \mathrm{n} 9 \mathrm{c}$ & $\mathrm{C} 44 \mathrm{H} 7308 \mathrm{NP}$ \\
\hline Ethanolamine & C18:0/C18:3n3 & C41H7508NP \\
\hline Ethanolamine & $\mathrm{C} 18: 2 \mathrm{n} 6 \mathrm{c} / \mathrm{C} 18 \mathrm{in} 9 \mathrm{c}$ & C41H7508NP \\
\hline Ethanolamine & $\mathrm{C} 18: 0 / \mathrm{C} 18: 2 \mathrm{n} 6 \mathrm{c}$ & C41H7708NP \\
\hline Ethanolamine & C18:1n9c/C18:1n9c & $\mathrm{C} 41 \mathrm{H} 7708 \mathrm{NP}$ \\
\hline Inositol & C16:0/C18:3n3 & $\mathrm{C} 43 \mathrm{H} 76013 \mathrm{P}$ \\
\hline Inositol & C16:0/C18:2n6c & $\mathrm{C} 43 \mathrm{H} 78013 \mathrm{P}$ \\
\hline Inositol & C16:0/C18:1n9c & $\mathrm{C} 43 \mathrm{H} 78013 \mathrm{P}$ \\
\hline Inositol & $\mathrm{C} 18: 2 \mathrm{n} 6 \mathrm{c} / \mathrm{C} 18: 3 \mathrm{n} 3$ & $\mathrm{C} 45 \mathrm{H} 76013 \mathrm{P}$ \\
\hline Inositol & $\mathrm{C} 18: 2 \mathrm{n} 6 \mathrm{c} / \mathrm{C} 18 \mathrm{2n} 6 \mathrm{c}$ & $\mathrm{C} 45 \mathrm{H} 78013 \mathrm{P}$ \\
\hline Inositol & C18:3n3/C18:1n9c & $\mathrm{C} 45 \mathrm{H} 78013 \mathrm{P}$ \\
\hline Inositol & C18:0/C18:3n3 & $\mathrm{C} 45 \mathrm{H} 80013 \mathrm{P}$ \\
\hline Inositol & C18:2n6c/C18:1n9c & C45H80013P \\
\hline Inositol & C18:0/C18:2n6c & C45H82O13P \\
\hline Inositol & C18:1n9c/C18:1n9c & $\mathrm{C} 45 \mathrm{H} 82 \mathrm{O} 13 \mathrm{P}$ \\
\hline Phosphatidic acid & $\mathrm{C} 16: 0 / \mathrm{C} 18: 1 \mathrm{n} 9 \mathrm{c}$ & C37H6807P \\
\hline Phosphatidic acid & C18:0/C18:3n3 & С39H6807P \\
\hline Phosphatidic acid & C18:2n6c/C18:1n9c & С39H6807P \\
\hline Lyso-Choline & C16:0 & $\mathrm{C} 24 \mathrm{H} 5007 \mathrm{NP}$ \\
\hline Lyso-Choline & $\mathrm{C} 18: 3 \mathrm{n} 3$ & $\mathrm{C} 26 \mathrm{H} 4807 \mathrm{NP}$ \\
\hline Lyso-Choline & $\mathrm{C} 18: 2 \mathrm{n} 6 \mathrm{c}$ & $\mathrm{C} 26 \mathrm{H} 5007 \mathrm{NP}$ \\
\hline Lyso-Choline & C18:1n9c & $\mathrm{C} 26 \mathrm{H} 52 \mathrm{O} 7 \mathrm{NP}$ \\
\hline Lyso-Choline & $\mathrm{C} 18: 0$ & C26H54O7NP \\
\hline Lyso-Ethanolamine & $\mathrm{C} 18: 2 \mathrm{n} 6 \mathrm{c}$ & $\mathrm{C} 23 \mathrm{H} 4307 \mathrm{NP}$ \\
\hline
\end{tabular}


Table 2.- Nomenclature of glycerol-containing liposomal suspensions.

\begin{tabular}{|c|c|c|c|}
\hline \multirow[t]{2}{*}{ Sonication conditions } & \multicolumn{3}{|c|}{ Raw materials } \\
\hline & SL & PW2 & PW5 \\
\hline $\begin{array}{c}\text { Method A: } \\
\text { Strong: } 120 \mathrm{~W}-5 \mathrm{~min}\end{array}$ & LSL & L2A & L5A \\
\hline $\begin{array}{c}\text { Method B: } \\
\text { Light: } 30 \mathrm{~W}-2 \mathrm{~min}\end{array}$ & & L2B & L5B \\
\hline $\begin{array}{c}\text { Method A, } \\
\text { without glycerol }\end{array}$ & & & L5Ang \\
\hline
\end{tabular}


Table 3.- Compositional properties of soy lecithin (SL), partially purified phosphatidylcholine after two acetone washing steps (PW2) and five acetone washing steps (PW5)

\begin{tabular}{|c|c|c|c|}
\hline & SL & PW2 & PW5 \\
\hline \multicolumn{4}{|l|}{ Proximate analysis (\%) } \\
\hline Crude lipid & $75.06 \pm 1.05^{a}$ & $68.55 \pm 1.95^{b}$ & $67.71 \pm 1.42^{b}$ \\
\hline Ash & $6.70 \pm 0.21^{\mathrm{a}}$ & $10.77 \pm 0.85^{b}$ & $10.05 \pm 0.10^{b}$ \\
\hline Moisture & $6.63 \pm 1.23^{a}$ & $5.72 \pm 0.98^{\mathrm{a}}$ & $5.43 \pm 1.11^{a}$ \\
\hline Protein & $0.28 \pm 0.01^{\mathrm{a}}$ & $0.29 \pm 0.01^{a}$ & $0.27 \pm 0.01^{\mathrm{a}}$ \\
\hline \multicolumn{4}{|l|}{ Amino acids $(\mathrm{mg} / 100 \mathrm{~g})$} \\
\hline Aspartic acid & $39.7 \pm 1.6^{\mathrm{a}}$ & $33.0 \pm 1.7^{b}$ & $31.3 \pm 1.3^{b}$ \\
\hline Threonine & $11.4 \pm 0.5^{\mathrm{a}}$ & $9.5 \pm 0.4^{b}$ & $8.8 \pm 0.4^{b}$ \\
\hline Serine & $25.2 \pm 1.3^{\mathrm{a}}$ & $20.6 \pm 0.8^{b}$ & $20.0 \pm 1.2^{\mathrm{b}}$ \\
\hline Glutamic acid & $58.4 \pm 2.8^{\mathrm{a}}$ & $51.8 \pm 2.2^{\mathrm{b}}$ & $47.0 \pm 2.5^{b}$ \\
\hline Glycine & $14.1 \pm 0.6^{\mathrm{a}}$ & $11.6 \pm 0.5^{\mathrm{b}}$ & $10.9 \pm 0.5^{b}$ \\
\hline Alanine & $15.9 \pm 0.8^{\mathrm{a}}$ & $13.2 \pm 0.7^{b}$ & $12.4 \pm 0.5^{b}$ \\
\hline Cysteine & $1.2 \pm 0.1^{\mathrm{a}, \mathrm{b}}$ & $1.0 \pm 0.1^{\mathrm{a}}$ & $1.3 \pm 0.1^{\mathrm{b}}$ \\
\hline Valine & $15.5 \pm 0.9^{\mathrm{a}}$ & $12.6 \pm 0.5^{\mathrm{b}}$ & $11.9 \pm 0.6^{\mathrm{b}}$ \\
\hline Methionine & $2.4 \pm 0.1^{a}$ & $2.0 \pm 0.1^{b}$ & $1.6 \pm 0.1^{c}$ \\
\hline Isoleucine & $9.6 \pm 0.4^{a}$ & $7.7 \pm 0.4^{b}$ & $7.3 \pm 0.3^{b}$ \\
\hline Leucine & $17.2 \pm 1.1^{\mathrm{a}}$ & $14.3 \pm 0.7^{b}$ & $13.4 \pm 0.7^{b}$ \\
\hline Tyrosine & $7.9 \pm 0.5^{a}$ & $6.8 \pm 0.4^{b}$ & $5.0 \pm 0.2^{c}$ \\
\hline Phenylalanine & $15.4 \pm 0.9^{\mathrm{a}}$ & $52.5 \pm 2.5^{b}$ & $48.4 \pm 2.8^{b}$ \\
\hline Histidine & $12.3 \pm 0.7^{\mathrm{a}}$ & $9.8 \pm 0.3^{b}$ & $7.7 \pm 0.4^{c}$ \\
\hline Lysine & $24.3 \pm 1.0^{\mathrm{a}}$ & $18.7 \pm 1.0^{b}$ & $17.2 \pm 1.0^{\mathrm{b}}$ \\
\hline Arginine & $21.0 \pm 1.0^{\mathrm{a}}$ & $17.1 \pm 1.0^{\mathrm{b}}$ & $16.6 \pm 0.9^{b}$ \\
\hline Proline & $12.0 \pm 0.6^{\mathrm{a}}$ & $10.2 \pm 0.5^{\mathrm{b}}$ & $9.7 \pm 0.5^{b}$ \\
\hline Tocopherols (mg/100 g) & $92.61 \pm 3.7^{a}$ & $5.80 \pm 0.15^{b}$ & $1.05 \pm 0.01^{c}$ \\
\hline$\nu$-tocopherol & $65.17 \pm 2.83^{a}$ & $3.93 \pm 0.13^{b}$ & $0.72 \pm 0.01^{c}$ \\
\hline$\delta$-tocopherol & $24.49 \pm 0.94^{\mathrm{a}}$ & $1.67 \pm 0.02^{b}$ & $0.28 \pm 0.00^{c}$ \\
\hline$\alpha$-tocopherol & $2.95 \pm 0.01^{\mathrm{a}}$ & $0.18 \pm 0.00^{b}$ & $0.04 \pm 0.00^{c}$ \\
\hline
\end{tabular}

Different letters $(a, b, c)$ indicate significant differences $(p \leq 0.05)$ among raw materials. 
Table 4. Fatty acid composition of lipid fractions (neutral lipids, free fatty acids and phospholipids) of soy lecithin (SL) and partially purified phosphatidylcholine after two acetone washing steps (PW2) and five acetone washing steps (PW5).

\begin{tabular}{|c|c|c|c|c|c|c|c|c|c|}
\hline & \multicolumn{3}{|c|}{$\begin{array}{c}\text { Neutral lipids (NL) } \\
\text { (mg/g sample) }\end{array}$} & \multicolumn{3}{|c|}{$\begin{array}{c}\text { Free Fatty Acids (FFA) } \\
\text { (mg/g sample) }\end{array}$} & \multicolumn{3}{|c|}{$\begin{array}{c}\text { Phospholipids (PL) } \\
\text { (mg/g sample) }\end{array}$} \\
\hline & SL & PW2 & PW5 & SL & PW2 & PW5 & SL & PW2 & PW5 \\
\hline C16:0 & $34.12 \pm 1.50^{\mathrm{a}}$ & $4.18 \pm 0.01^{b}$ & $3.67 \pm 0.39^{c}$ & $5.35 \pm 0.22^{a}$ & $1.91 \pm 0.14^{b}$ & $1.72 \pm 0.09^{c}$ & $88.40 \pm 0.04^{a}$ & $97.72 \pm 22.88^{\mathrm{a}}$ & $92.38 \pm 20.76^{a}$ \\
\hline C18:0 & $13.37 \pm 0.61^{a}$ & $1.23 \pm 0.02^{b}$ & $0.96 \pm 0.04^{c}$ & $2.50 \pm 0.07^{a}$ & $1.24 \pm 0.15^{b}$ & $1.23 \pm 0.05^{b}$ & $20.10 \pm 0.01^{a}$ & $22.15 \pm 5.64^{\mathrm{a}}$ & $20.91 \pm 4.65^{a}$ \\
\hline C18:1n9c & $56.20 \pm 2.77^{\mathrm{a}}$ & $2.49 \pm 0.14^{b}$ & $1.41 \pm 0.03^{c}$ & $6.03 \pm 0.12^{a}$ & $1.20 \pm 0.09^{b}$ & $0.64 \pm 0.30^{c}$ & $36.84 \pm 0.51^{\mathrm{a}}$ & $40.74 \pm 10.11^{a}$ & $38.14 \pm 9.01^{\mathrm{a}}$ \\
\hline $\mathrm{C} 18: 2 \mathrm{n} 6 \mathrm{c}$ & $162.50 \pm 6.94^{\mathrm{a}}$ & $10.73 \pm 0.72^{b}$ & $8.26 \pm 0.12^{c}$ & $16.28 \pm 0.65^{a}$ & $1.56 \pm 0.24^{b}$ & $0.56 \pm 0.31^{c}$ & $259.86 \pm 4.89^{a}$ & $287.95 \pm 71.42^{\mathrm{a}}$ & $267.64 \pm 62.81^{a}$ \\
\hline$C 18: 3 n 3$ & $26.25 \pm 1.18^{\mathrm{a}}$ & $1.58 \pm 0.09^{b}$ & $1.18 \pm 0.01^{\mathrm{c}}$ & $1.23 \pm 0.02^{\mathrm{a}}$ & $0.13 \pm 0.01^{b}$ & $0.07 \pm 0.01^{c}$ & $33.69 \pm 0.67^{a}$ & $37.18 \pm 9.18^{a}$ & $34.20 \pm 8.09^{\mathrm{a}}$ \\
\hline SFA & $47.50 \pm 2.11^{a}$ & $5.41 \pm 0.03^{b}$ & $4.63 \pm 0.43^{c}$ & $7.85 \pm 0.29^{a}$ & $3.15 \pm 0.29^{b}$ & $2.95 \pm 0.14^{b}$ & $108.50 \pm 0.05^{a}$ & $119.87 \pm 28.52^{\mathrm{a}}$ & $113.29 \pm 25.41^{a}$ \\
\hline MUFA+PUFA & $244.95 \pm 10.89^{a}$ & $14.79 \pm 0.95^{b}$ & $10.85 \pm 0.14^{c}$ & $23.53 \pm 0.79^{a}$ & $2.89 \pm 0.34^{b}$ & $1.27 \pm 0.62^{c}$ & $330.39 \pm 6.08^{a}$ & $365.88 \pm 90.71^{a}$ & $339.99 \pm 79.92^{a}$ \\
\hline
\end{tabular}

SFA: sum of saturated fatty acids; MUFA+PUFA sum of monounsaturated and polyunsaturated fatty acids. Different letters $(a, b, c)$ indicate significant differences $(p \leq 0.05)$ among raw materials for each lipid fraction. 
Table 5. Size (z-average), polydispersity and zeta potential of fresh liposomal dispersions.

\begin{tabular}{lccc}
\hline & $\begin{array}{c}\text { Size } \\
(\mathrm{nm})\end{array}$ & $\begin{array}{c}\text { Polydispersity } \\
\text { PDI }\end{array}$ & $\begin{array}{c}\text { Z potential } \\
(\mathrm{mV})\end{array}$ \\
\cline { 2 - 4 } LSL & $132 \pm 0.59^{\mathrm{a}}$ & $0.22 \pm 0.01^{\mathrm{ab}}$ & $-42.62 \pm 2.05^{\mathrm{ab}}$ \\
L2A & $108 \pm 0.35^{\mathrm{b}}$ & $0.17 \pm 0.01^{\mathrm{a}}$ & $-44.30 \pm 4.52^{\mathrm{b}}$ \\
L2B & $102 \pm 0.45^{\mathrm{c}}$ & $0.24 \pm 0.02^{\mathrm{b}}$ & $-41.53 \pm 2.84^{\mathrm{ab}}$ \\
L5A & $87 \pm 0.66^{\mathrm{d}}$ & $0.24 \pm 0.01^{\mathrm{bc}}$ & $-36.23 \pm 1.67^{\mathrm{ac}}$ \\
L5B & $101 \pm 0.52^{\mathrm{c}}$ & $0.24 \pm 0.01^{\mathrm{bc}}$ & $-42.10 \pm 1.32^{\mathrm{ab}}$ \\
L5Ang & $74 \pm 0.31^{\mathrm{e}}$ & $0.27 \pm 0.01^{\mathrm{c}}$ & $-34.47 \pm 1.93^{\mathrm{c}}$ \\
\hline
\end{tabular}

Different letters $(a, b, c)$ indicate significant differences $(p \leq 0.05)$ among samples. 
Table 6. Melting temperature $(\mathrm{Tm})$ and Enthalpy change $(\Delta \mathrm{H})$ of fresh liposomal dispersions.

\begin{tabular}{ccccc} 
& $\mathrm{T}_{\text {peak1 }}\left({ }^{\circ} \mathrm{C}\right)$ & $\Delta \mathrm{H}_{\text {peak1 }}(\mathrm{J} / \mathrm{g})$ & $\mathrm{T}_{\text {peak2 }}\left({ }^{\circ} \mathrm{C}\right)$ & $\Delta \mathrm{H}_{\text {peak2 }}(\mathrm{J} / \mathrm{g})$ \\
\cline { 2 - 5 } LSL & $-6.3 \pm 0.25^{\mathrm{a}}$ & $76.2 \pm 3.81^{\mathrm{a}}$ & $9.0 \pm 0.54^{\mathrm{a}}$ & $4.42 \pm 0.81^{\mathrm{ab}}$ \\
L2A & $-7.8 \pm 0.31^{\mathrm{b}}$ & $72.7 \pm 2.18^{\mathrm{b}}$ & $9.2 \pm 0.37^{\mathrm{a}}$ & $6.81 \pm 1.02^{\mathrm{b}}$ \\
L2B & $-6.2 \pm 0.19^{\mathrm{a}}$ & $67.5 \pm 4.05^{\mathrm{b}}$ & $7.9 \pm 0.32^{\mathrm{bc}}$ & $3.15 \pm 0.28^{\mathrm{a}}$ \\
L5A & $-8.9 \pm 0.27^{\mathrm{c}}$ & $48.1 \pm 1.92^{\mathrm{c}}$ & $11.3 \pm 0.45^{\mathrm{d}}$ & $8.34 \pm 0.67^{\mathrm{d}}$ \\
L5B & $-7.0 \pm 0.18^{\mathrm{d}}$ & $74.2 \pm 2.37^{\mathrm{ab}}$ & $9.0 \pm 0.36^{\mathrm{a}}$ & $5.04 \pm 1.01^{\mathrm{ab}}$ \\
L5Ang & $-1.7 \pm 0.09^{\mathrm{f}}$ & $106.2 \pm 6.37^{\mathrm{d}}$ & $8.4 \pm 0.50^{\mathrm{b}}$ & $2.20 \pm 0.13^{\mathrm{c}}$
\end{tabular}

Different letters $(a, b, c)$ indicate significant differences $(p \leq 0.05)$ among samples. 
Effect of chemical composition and sonication procedure on properties of food-grade soy lecithin liposomes with added glycerol.

D. Taladrid, D. Marín, A. Alemán, I. Álvarez-Acero, P. Montero, M.C. Gómez-Guillén

Acetone-washing

SOY LECITHIN

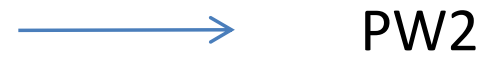

2-steps
Acetone-washing

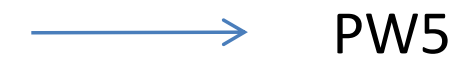

5-steps

\section{ULTRASONICATION}

A: $90 \%$ amplitude, 5 min $\downarrow$ B: $20 \%$ amplitude, 2 min

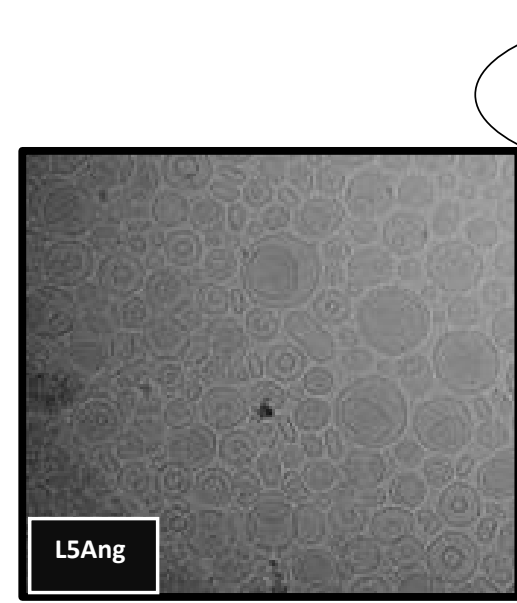

Without glycerol

\section{LIPOSOMES $\leftarrow$ Glycerol}

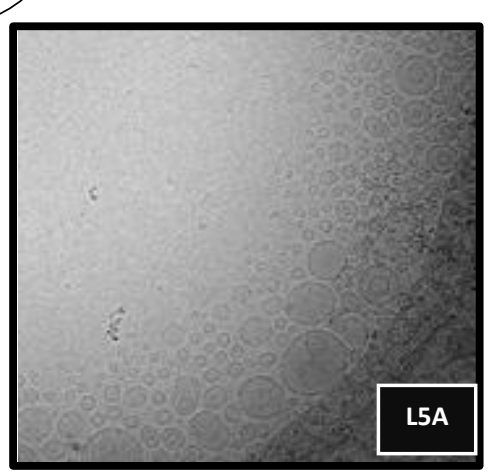

With glycerol
Chemical composition
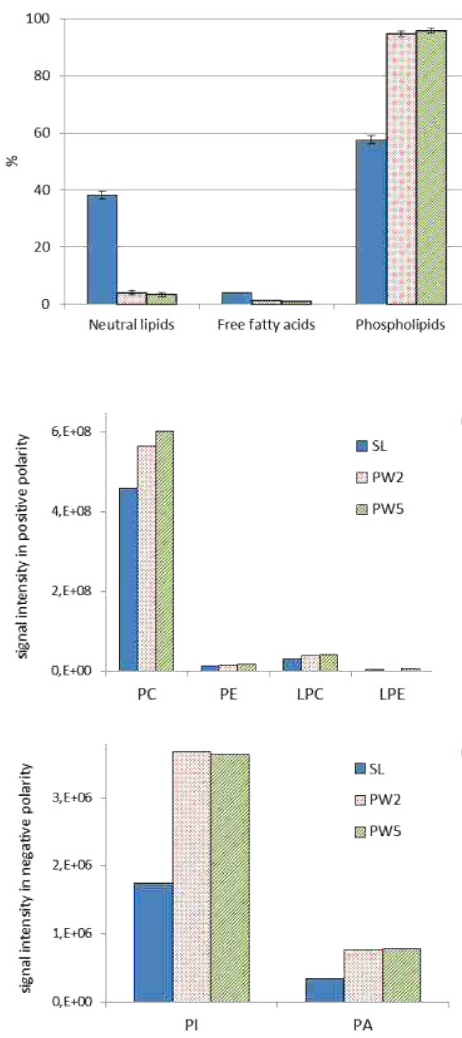